\title{
A New One-parameter G Family of Compound Distributions:Copulas, Statistical Properties and Applications
}

\author{
Mohamed Aboraya* \\ Department of Applied, Mathematical and Actuarial Statistics, Faculty of Commerce, Damietta University, Damietta, Egypt
}

\begin{abstract}
This work introduces a new one-parameter compound G family. Relevant statistical properties are derived. The new density can be "asymmetric right skewed with one peak and a heavy tail", "symmetric" and "left skewed with one peak". The new hazard function can be "upside-down", "upside-down-constant", "increasing", "decreasing" and "decreasing-constant". Many bivariate types have been also derived via different common copulas. The estimation of the model parameters is performed by maximum likelihood method. The usefulness and flexibility of the new family is illustrated by means of two real data sets.
\end{abstract}

Keywords Poisson Family, Compound Models, Moments, Farlie Gumbel Morgenstern Copula, Ali-Mikhail-Haq Copula.

DOI:10.19139/soic-2310-5070-1239

\section{Introduction and motivation}

In the last few years, huge efforts have been paid to derive many new $\mathrm{G}$ families using the well knowm methods. These new $\mathrm{G}$ families have been used for modeling non-censored and censored real data sets in many applied studies such as finance, econometrics, value at risk applications, insurance, biology, engineering, forecasting, medicine and environmental sciences see, for example, Marshall and Olkin (1997) (Marshall-Olkin-G (MO-G) family), Eugene et al. (2002) (beta generalized-G (B-G) family), Yousof et al. (2015) (transmuted exponentiated generalized (TEG) family), Rezaei et al. (2017) (Topp Leone generated (TLG) family), Merovci et al. (2017) (exponentiated transmuted-G (ET-G) family), Aryal and Yousof (2017) (exponentiated generalized-G Poisson (EGGP) family), Brito et al. (2017) (Topp-Leone odd log-logistic-G (TLOLL-G) family), Yousof et al. (2017a) (Burr X G (BX-G) family), Hamedani et al. (2017) (type I general exponential-G (TIGE-G) family), Korkmaz et al. (2018a) (exponential Lindley odd log-logistic-G (ELOLL-G) family), Cordeiro et al. (2018) (Burr XII-G (BXII-G) family), Hamedani et al. (2018) (extended-G (Ex-G) family), Korkmaz et al. (2018b) (Marshall-Olkin generalized-G Poisson (MOGGP) family), Yousof et al. (2018) (Burr-Hatke-G (BH-G) family), Nascimento et al. (2019) (Nadarajah-Haghighi-G (NH-G) family), Hamedani et al. (2019) ( type II general exponential-G (TIIGEG) family), Yousof et al. (2020) (Weibull G Poisson (WGP) family), Merovci et al. (2020) (Poisson Topp Leone G (PTL-G) family), Karamikabir et al. (2020) (Weibull Topp-Leone generated (WTL-G) family), Korkmaz et al. (2020) (Hjorth-G (Hj-G) family), Alizadeh et al. (2020a) (flexible Weibull generated (FWG) family), Alizadeh et al. (2020b) (transmuted odd log-logistic-G (TOLL-G) family), El-Morshedy et al. (2021) (Poisson generalized exponential G (PGE-G) family) and Altun et al. (2021) (Gudermannian generated-G (GG-G) family), among others. In this paper we propose and study a new family of distributions using the zero truncated Poisson (ZTP) distribution with a strong physical motivation. Suppose that a system has $N$ subsystems functioning independently

\footnotetext{
*Correspondence to: Mohamed Aboraya (mohamedaboraya17@gmail.com).Department of Applied, Mathematical and Actuarial Statistics, Faculty of Commerce, Damietta University, Damietta, Egypt.
}

ISSN 2310-5070 (online) ISSN 2311-004X (print)

Copyright $(02021$ International Academic Press 
at a given time where $N$ has ZTP distribution with parameter $\zeta$. It is the conditional probability distribution of a Poisson-distributed random variable (R.V), given that the value of the R.V is not zero (see maurya and Nadarajah (1998)). The probability mass function (PMF) of $N$ is given by

$$
P(N=n)=\left[\zeta^{n} \exp (-\zeta)\right] /\left.\left(n ! \zeta_{\bullet}\right)\right|_{(n=1,2, \ldots)},
$$

where $\zeta_{\bullet}=1-\exp (-\zeta)$. Note that for ZTP R.V, the expected value $\mathbf{E}(N \mid \zeta)$ and variance $\operatorname{Var}(N \mid \zeta)$ are, respectively, given by

$$
\mathbf{E}(N \mid \zeta)=\zeta \zeta_{\bullet}^{-1}
$$

and

$$
\operatorname{Var}(N \mid \zeta)=\frac{1}{\zeta_{\bullet}}\left(\zeta+\zeta^{2}\right)-\frac{1}{\zeta_{\bullet}^{2}} \zeta^{2}
$$

Suppose that the failure time of each subsystem has the reciprocal Rayleigh G ("RR-G(P)" for short) family defined by the cumulative distribution function (CDF) and probability density function(PDF) given by

$$
H_{\underline{\mathbf{P}}}(x)=\left.\exp \left(-\frac{1}{\nabla_{x, \underline{\mathbf{P}}}^{2}}\right)\right|_{x \in \mathbb{R}} .
$$

and

$$
h_{\underline{\mathbf{P}}}(x)=2 g_{\underline{\mathbf{P}}}(x) \frac{\bar{G}_{\underline{\mathbf{P}}}(x)}{G_{\underline{\mathbf{P}}}(x)^{3}} \exp \left(-\frac{1}{\nabla_{x, \underline{\mathbf{P}}}^{2}}\right), x \in \mathbb{R},
$$

respectively, where

$$
\nabla_{x, \underline{\mathbf{P}}}=\left.\frac{G_{\underline{\mathbf{P}}}(x)}{\bar{G}_{\underline{\mathbf{P}}}(x)}\right|_{x \in \mathbb{R}},
$$

and $\underline{\mathbf{P}}$ is the parameter vector of the baseline model and $\bar{G}_{\mathbf{P}}(x)=1-G_{\mathbf{P}}(x)$ is the survival function of the baseline model. Let $Y_{i}$ denote the failure time of the ith subsystem and let $X=\min \left\{Y_{1}, Y_{2}, \cdots, Y_{N}\right\}$. Due to Aryal and Yousof (2017), Korkmaz et al. (2018), Yousof et al. (2018a), Abouelmagd et al. (2019), et al. (2019) and Yousof et al. (2020), the conditional CDF of $X$ given $N$ is

$$
F(x \mid N)=1-\operatorname{Pr}(X>x \mid N)=1-\left[1-H_{\underline{\mathbf{P}}}(x)\right]^{N} .
$$

Therefore, the CDF of the Poisson reciprocal Rayleigh (PRR-G) family can be expressed as

$$
F_{\underline{\Phi}}(x)=\left.\zeta_{\bullet}^{-1}\left\{1-\exp \left[-\zeta \exp \left(-\frac{1}{\nabla_{x, \underline{\mathbf{P}}}^{2}}\right)\right]\right\}\right|_{x \in \mathbb{R}},
$$

where $\underline{\boldsymbol{\Omega}}=(\zeta, \underline{\mathbf{P}})$. The corresponding PDF as

$$
f_{\underline{\Phi}}(x)=\left.2 \zeta \frac{g_{\underline{\mathbf{P}}}(x) \bar{G}_{\underline{\mathbf{P}}}(x)}{\zeta_{\bullet} G_{\underline{\mathbf{P}}}(x)^{3}} \exp \left(-\frac{1}{\nabla_{x, \underline{\mathbf{P}}}^{2}}\right) \exp \left[-\zeta \exp \left(-\frac{1}{\nabla_{x, \underline{\mathbf{P}}}^{2}}\right)\right]\right|_{x \in \mathbb{R}} .
$$

In this work, a special attention is paid to two special members called the Poisson reciprocal Rayleigh exponential and the Poisson reciprocal Rayleigh Fréchet distributions. The new density of the Poisson reciprocal Rayleigh exponential model can be "asymmetric right skewed with one peak and a heavy tail", "symmetric" and "left skewed with one peak". The new hazard rate function of the can be Poisson reciprocal Rayleigh exponential model "upside-down-constant", "increasing" and "decreasing-constant". The new density of the Poisson reciprocal Rayleigh Fréchet model can be "asymmetric right skewed with one peak and a heavy tail", "symmetric" and "left skewed with one peak". The new hazard rate function of the Poisson reciprocal Rayleigh Fréchet model can be "upside-down", "increasing" and "decreasing". So, the family may be useful in modeling the "asymmetric right skewed with one peak and a heavy tail" real data sets, "symmetric" real data setsand "left skewed with one peak" 
real data sets. Also, the family may be useful in modeling the real data sets which have "upside-down", "upsidedown-constant", "increasing", "decreasing" and "decreasing-constant" hazard rate functions.

The new family is better than the odd Lindley family, Marshall-Olkin family, the Burr-Hatke family, generalized Marshall-Olkin family, Beta family, Marshall-Olkin Kumaraswamy family, Kumaraswamy family, the Burr X family and Kumaraswamy Marshall-Olkin family in modeling the bimodal right skewed relief times data set so the new family could be considered as a good alternative to these families. The new family is better than the Marshal-Olkin family, Generalized Marshal-Olkin family, Kumaraswamy family, beta family, Kumaraswamy Marshal-Olkin family and Marshal-Olkin Kumaraswamy family in modeling the gauge lengths data set so the new family could be considered as a good alternative to these families.

\section{Mathematical properties}

\subsection{Useful expansions}

Using the power series

$$
\exp \left(v_{1}\right)=\sum_{v_{2}=0}^{+\infty} \frac{1}{v_{2} !} v_{1}^{v_{2}}
$$

the PDF in (6) can be written as

$$
f_{\underline{\mathbf{\Phi}}}(x)=2 \sum_{h=0}^{+\infty} \frac{\zeta^{1+h}(-1)^{h}}{h ! \zeta_{\bullet}} \frac{g_{\underline{\mathbf{P}}}(x) \bar{G}_{\underline{\mathbf{P}}}(x)}{G_{\underline{\mathbf{P}}}(x)^{3}} \exp \left[-(1+h) \frac{1}{\nabla_{x, \underline{\mathbf{P}}}^{2}}\right],
$$

again applying (7) to (8) we get

$$
f_{\underline{\Phi}}(x)=2 \sum_{h, v=0}^{+\infty} \frac{\zeta^{1+h}(-1)^{h+v}(1+h)^{v}}{h ! v ! \zeta \bullet} g_{\underline{\mathbf{P}}}(x) \bar{G}_{\underline{\mathbf{P}}}(x)^{1+2 v} G_{\underline{\mathbf{P}}}(x)^{-2 v-3} .
$$

If $\left|\frac{v_{1}}{v_{2}}\right|<1$ and $v_{3}>0$ is a real non-integer, the following power series holds

$$
\left(1-\frac{v_{1}}{v_{2}}\right)^{v_{3}-1}=\sum_{l=0}^{+\infty} \frac{(-1)^{l} \Gamma\left(v_{3}\right)}{i ! \Gamma\left(v_{3}-l\right)}\left(\frac{v_{1}}{v_{2}}\right)^{l}
$$

Applying (10) to (9) we have

$$
f_{\underline{\Phi}}(x)=2 \sum_{h, v, l=0}^{+\infty} \frac{\zeta^{1+h}(-1)^{h+v+l}(1+h)^{v} \Gamma(2+2 v) \mathbf{c}^{*}}{h ! v ! l ! \zeta \bullet \Gamma(2+2 v-l) \mathbf{c}^{*}} g_{\underline{\mathbf{P}}}(x) G_{\underline{\mathbf{P}}}(x)^{l-2 v-3},
$$

where $\mathbf{c}^{*}=l-2(v+1)$, then

$$
f_{\underline{\Phi}}(x)=\sum_{v, l=0}^{+\infty} \boldsymbol{\Upsilon}_{v, l} \pi_{\mathbf{c}^{*}}(x ; \underline{\mathbf{P}}),
$$

where

$$
\mathbf{\Upsilon}_{v, l}=\sum_{h=0}^{+\infty} \frac{2 \zeta^{1+h}(-1)^{h+v+l}(1+h)^{v} \Gamma(2+2 v)}{h ! v ! l ! \zeta_{\bullet} \Gamma(2+2 v-l)[l-2(v+1)]},
$$

and $\pi_{\mathbf{c}^{*}}(x ; \underline{\mathbf{P}})=\mathbf{c}^{*} g_{\underline{\mathbf{P}}}(x)\left[G_{\underline{\mathbf{P}}}(x)\right]^{\mathbf{c}^{*}-1}$ is the PDF of the exponentiated-G (exp-G) family with power parameter $\mathbf{c}^{*}$. Equation (12) reveals that the density of $X$ can be expressed as a linear mixture of exp-G densities. So, several mathematical properties of the new family can be obtained from those of the exp-G distribution. Similarly, the CDF 
of the PRR-G family can also be expressed as a mixture of exp-G CDFs given by

$$
F(x)=\sum_{v, l=0}^{+\infty} \mathbf{\Upsilon}_{v, l} \Pi_{\mathbf{c}^{*}}(x ; \underline{\mathbf{P}}),
$$

where $\Pi_{\mathbf{c}^{*}}(x ; \underline{\mathbf{P}})=G_{\underline{\mathbf{P}}}(x)^{\mathbf{c}^{*}}$ is the CDF of the exp-G family with power parameter $\mathbf{c}^{*}$.

\subsection{Quantile function $(Q F)$ and random number generation}

The QF of the R.V $X$, where $X \sim \operatorname{PRR}-\mathrm{G}(\zeta, \underline{\mathbf{P}})$, is obtained by inverting its CDF in (5) as

$$
\left.Q(u)=G^{-1}\left[1+\sqrt{-\log \left\{-\frac{1}{\zeta} \log \left(1+u \zeta_{\bullet}\right)\right.}\right\}\right]^{-1}, 0 \leq u \leq 1,
$$

Simulating the PRR-G R.V is straightforward. If $U$ is a uniform variate on the unit interval $(0,1)$, then the R.V $X=Q(U)$ follows (6).

\subsection{Moments}

Let $Y_{\mathbf{c}^{*}}$ be a R.V having density $\pi_{\mathbf{c}^{*}}(x ; \underline{\mathbf{P}})$. The $r$ th ordinary moment of $X$, say $\mu_{r, X}^{\prime}$, follows from (12) as

$$
\mu_{r, X}^{\prime}=\mathbf{E}\left(X^{r}\right)=\sum_{v, l=0}^{+\infty} \Upsilon_{v, l} \mathbf{E}\left(Y_{\mathbf{c}^{*}}^{r}\right)
$$

where

$$
\mathbf{E}\left(Y_{\zeta}^{r}\right)=\zeta \int_{-\infty}^{+\infty} x^{r} g_{\underline{\mathbf{P}}}(x) G_{\underline{\mathbf{P}}}(x)^{\zeta-1} d x,
$$

can be evaluated numerically in terms of the baseline qf $Q_{G}(u)=G^{-1}(u)$ as

$$
\mathbf{E}\left(Y_{\zeta}^{r}\right)=\zeta \int_{0}^{1} u^{\zeta-1}\left[Q_{G}(u)\right]^{r} d u
$$

Setting $r=1$ in (14) gives the mean of $X$.

\subsection{Incomplete moments and mean deviations}

The $r$ th incomplete moment of $X$ is defined by $\omega_{r, X}(y)=\int_{-\infty}^{y} x^{r} f_{\underline{\Phi}}(x) d x$. We can write from (12)

$$
\omega_{r, X}(y)=\sum_{v, l=0}^{+\infty} \mathbf{\Upsilon}_{v, l} \omega_{r, \mathbf{c}^{*}}(y)
$$

where

$$
\omega_{r, \zeta}(y)=\int_{0}^{G(y)} u^{\zeta-1}\left[Q_{G}(u)\right]^{r} d u .
$$

The integral $m_{r, \zeta}(y)$ can be determined analytically for any special model with closed-form expressions for the $Q_{G}(u)$ or computed at least numerically for most baseline distributions. Two important applications of the first incomplete moment are related to the mean deviations about the mean and median and to the Bonferroni and Lorenz curves. 


\subsection{Moment generating function (MGF)}

The MGF of $X$, say $M_{X}(t)=\mathbf{E}(\exp (t X))$, is obtained from (12) as

$$
M_{X}(t)=\sum_{v, l=0}^{+\infty} \Upsilon_{v, l} M_{\mathbf{c}^{*}}(t),
$$

where $M_{\mathbf{c}^{*}}(t)$ is the generating function of $Y_{\mathbf{c}^{*}}$ given by

$$
M_{\zeta}(t)=\zeta \mathbf{I}_{-\infty}^{+\infty}(x)=\zeta \mathbf{I}_{0}^{+1}(u)
$$

where

$$
\mathbf{I}_{-\infty}^{+\infty}(x)=\int_{-\infty}^{+\infty} \exp (t x) g_{\underline{\mathbf{P}}}(x)\left[G_{\underline{\mathbf{P}}}(x)\right]^{\zeta-1} d x
$$

and

$$
\mathbf{I}_{0}^{+1}(u)=\int_{0}^{1} \exp \left[t Q_{G}(u ; \zeta)\right] u^{\zeta-1} d u
$$

The two integrals $\mathbf{I}_{-\infty}^{+\infty}(x)$ and $\mathbf{I}_{0}^{+1}(u)$ can be computed numerically for most base line distributions.

\section{Copulas}

A copula is a multivariate CDF for which the marginal distribution of each R.V is uniform on the interval $[0,1]$. Copulas are used to describe the dependence between R.Vs. In this Section, we derive some new bivariate PRR (B-PRR) type distributions using the Farlie Gumbel Morgenstern (FGM) copula (Morgenstern (1956), Gumbel (1958), Gumbel (1960), Johnson and Kotz (1975) and Johnson and Kotz (1977)), the modified version of the FGM copula (Rodriguez-Lallena and Ubeda-Flores (2004)), the Clayton copula, the Renyi's copula (Pougaza and Djafari (2011)) and finally with the Ali-Mikhail-Haq copula (Ali et al. (1987)). The Multivariate PRR (M-PRR) type is also presented under the Clayton copula. However, many future works could allocated to the study these new models (see also Al-babtain et al. (2020), Elgohari et al. (2020a,b), Mansour et al. (2020a-f), Ibrahim et al. (2021) and Ali et al. (2021a,b) as examples).

\subsection{FGM copula}

Consider the joint CDF (J-CDF) of the FGM family with dependence parameter $\varsigma$, where

$$
\mathfrak{C}_{\varsigma}(v, \mathfrak{a})=\left.v \mathfrak{a}\left(1+\varsigma v^{\bullet} \mathfrak{a}^{\bullet}\right)\right|_{v \bullet=1-v, \mathfrak{a} \bullet=1-\mathfrak{a}},
$$

and the two marginal function $v=F_{1}, \mathfrak{a}=F_{2}, \varsigma \in(-1,1)$ and for every $v, \mathfrak{a} \in(0,1), \mathfrak{C}(v, 0)=\mathfrak{C}(0, \mathfrak{a})=0$ which is "grounded-minimum" and $\mathfrak{C}(v, 1)=v$ and $\mathfrak{C}(1, \mathfrak{a})=\mathfrak{a}$ which are "grounded-maximum". Then,

$$
\mathfrak{C}\left(v_{1}, \mathfrak{a}_{1}\right)+\mathfrak{C}\left(v_{2}, \mathfrak{a}_{2}\right)-\mathfrak{C}\left(v_{1}, \mathfrak{a}_{2}\right)-\mathfrak{C}\left(v_{2}, \mathfrak{a}_{1}\right) \geq 0 .
$$

A copula is continuous in $v$ and $\mathfrak{a}$; actually, it satisfies the stronger Lipschitz condition, where

$$
\left|\mathfrak{C}\left(v_{2}, \mathfrak{a}_{2}\right)-\mathfrak{C}\left(v_{1}, \mathfrak{a}_{1}\right)\right| \leq\left|v_{2}-v_{1}\right|+\left|\mathfrak{a}_{2}-\mathfrak{a}_{1}\right| .
$$

For $0 \leq v_{1} \leq v_{2} \leq 1$ and $0 \leq \mathfrak{a}_{1} \leq \mathfrak{a}_{2} \leq 1$, we have

$$
\operatorname{Pr}\left(v_{1} \leq v \leq v_{2}, \mathfrak{a}_{1} \leq \mathfrak{a} \leq \mathfrak{a}_{2}\right)=\mathfrak{C}\left(v_{1}, \mathfrak{a}_{1}\right)+\mathfrak{C}\left(v_{2}, \mathfrak{a}_{2}\right)-\mathfrak{C}\left(v_{1}, \mathfrak{a}_{2}\right)-\mathfrak{C}\left(v_{2}, \mathfrak{a}_{1}\right) \geq 0 .
$$


Then, setting $v^{\bullet}=1-\left.F\left(z_{1}\right)\right|_{\left[v^{\bullet}=(1-v) \in(0,1)\right]}$ and $\mathfrak{a}^{\bullet}=1-\left.F\left(z_{2}\right)\right|_{[\mathfrak{a} \bullet=(1-\mathfrak{a}) \in(0,1)]}$, we can esaily obtain the J-CDF of the PRR using the FGM family

$$
\begin{aligned}
\mathfrak{C}_{\varsigma}\left(z_{1}, z_{2}\right)= & \zeta_{\bullet 1}^{-1}\left[1-\exp \left(-\zeta_{1} \varrho_{\underline{\mathbf{P}}}\left(z_{1}\right)\right)\right] \\
& \times \zeta_{\bullet 2}^{-1}\left[1-\exp \left(-\zeta_{2} \varrho_{\underline{\mathbf{P}}}\left(z_{2}\right)\right)\right] \\
& \times\left[1+\varsigma\left(\begin{array}{c}
\left\{1-\zeta_{\bullet 1}^{-1}\left[1-\exp \left(-\zeta_{1} \varrho_{\mathbf{P}}\left(z_{1}\right)\right)\right]\right\} \\
\times\left\{1-\zeta_{\bullet 2}^{-1}\left[1-\exp \left(-\zeta_{2} \varrho_{\underline{\mathbf{P}}}\left(z_{2}\right)\right)\right]\right\}
\end{array}\right)\right],
\end{aligned}
$$

where

$$
\varrho_{\underline{\mathbf{P}}}(z)=\exp \left(-\frac{1}{\nabla_{x, \underline{\mathbf{P}}}^{2}}\right) .
$$

The joint PDF can then be derived from $c_{\varsigma}(v, \mathfrak{a})=1+\left.\varsigma v^{\bullet} \mathfrak{a} \bullet\right|_{(v \bullet=1-2 v}$ and $\left.\mathfrak{a} \bullet=1-2 \mathfrak{a}\right)$ or from

$$
c_{\varsigma}\left(z_{1}, z_{2}\right)=f\left(z_{1}, z_{2}\right)=\mathfrak{C}\left(F_{1}, F_{2}\right) f_{1} f_{2} .
$$

\subsection{Modified FGM copula}

The modified FGM copula is defined as $\mathfrak{C}_{\varsigma}(v, \mathfrak{a})=\left.v \mathfrak{a}[1+\varsigma A(v) C(\mathfrak{a})]\right|_{\varsigma \in(-1,1)}$ or $\quad \mathfrak{C}_{\varsigma}(v, \mathfrak{a})=v \mathfrak{a}+$ $\left.\varsigma \widetilde{A}_{v} \widetilde{C}_{\mathfrak{a}}\right|_{\varsigma \in(-1,1)}$, where $\widetilde{A}_{v}=v A(v)$, and $\widetilde{C}_{\mathfrak{a}}=\mathfrak{a} C(\mathfrak{a})$ and $A(v)$ and $C(\mathfrak{a})$ are two continuous functions on $(0,1)$ with $A(0)=A(1)=C(0)=C(1)=0$. Let

$$
\begin{aligned}
& a_{1}(v)=\left.\inf \left\{\widetilde{A}_{v}: \frac{\partial}{\partial v} \widetilde{A}_{v}\right\}\right|_{\varpi_{1, v}}<0, \\
& a_{2}(v)=\left.\sup \left\{\widetilde{A}_{v}: \frac{\partial}{\partial v} \widetilde{A}_{v}\right\}\right|_{\varpi_{1, v}}<0, \\
& c_{1}(\mathfrak{a})=\left.\inf \left\{\widetilde{C}_{\mathfrak{a}}: \frac{\partial}{\partial \mathfrak{a}} \widetilde{C}_{\mathfrak{a}}\right\}\right|_{\varpi_{2, \mathfrak{a}}}>0, \\
& c_{2}(\mathfrak{a})=\left.\sup \left\{\widetilde{C}_{\mathfrak{a}}: \frac{\partial}{\partial \mathfrak{a}} \widetilde{C}_{\mathfrak{a}}\right\}\right|_{\varpi_{2, \mathfrak{a}}}>0 .
\end{aligned}
$$

Then,

$$
1 \leq \min \left\{a_{1}(v) a_{2}(v), c_{1}(\mathfrak{a}) c_{2}(\mathfrak{a})\right\}<+\infty,
$$

where

and

$$
\begin{gathered}
0=\frac{\partial}{\partial v} \widetilde{A}_{v}-v \frac{\partial}{\partial v} A(v)-A(v), \\
\varpi_{1, v}=\left\{v:\left.v \in(0,1)\right|_{\frac{\partial}{\partial v}} \widetilde{A}_{v} \text { exists }\right\},
\end{gathered}
$$

$$
\varpi_{2, \mathfrak{a}}=\left\{\mathfrak{a}:\left.\mathfrak{a} \in(0,1)\right|_{\frac{\partial}{\partial \mathfrak{a}}} \widetilde{C}_{\mathfrak{a}} \text { exists }\right\}
$$

3.2.1. Modified FGM type-I Consider the following functional form for both $A(v)$ and $C(\mathfrak{a})$. Then, the B-PRRFGM (Type-I) can be derived from

$$
\begin{aligned}
\mathfrak{C}_{\varsigma}\left(z_{1}, z_{2}\right)= & \zeta_{\bullet 1}^{-1}\left[1-\exp \left(-\zeta_{1} \varrho_{\underline{\mathbf{P}}}\left(z_{1}\right)\right)\right] \\
& \times \zeta_{\bullet 2}^{-1}\left[1-\exp \left(-\zeta_{2} \varrho_{\underline{\mathbf{P}}}\left(z_{2}\right)\right)\right] \\
& +\left.\varsigma\left(\begin{array}{c}
\zeta_{\bullet 1}^{-1}\left[1-\exp \left(-\zeta_{1} \varrho_{\underline{\mathbf{P}}}\left(z_{1}\right)\right)\right] \\
\times\left\{1-\zeta_{\bullet 1}^{-1}\left[1-\exp \left(-\zeta_{1} \varrho_{\mathbf{P}}\left(z_{1}\right)\right)\right]\right\} \\
\times \zeta_{\bullet 2}^{-1}\left[1-\exp \left(-\zeta_{2} \varrho_{\underline{\mathbf{P}}}\left(z_{2}\right)\right)\right] \\
\times\left\{1-\zeta_{\bullet 2}^{-1}\left[1-\exp \left(-\zeta_{2} \varrho_{\mathbf{P}}\left(z_{2}\right)\right)\right]\right\}
\end{array}\right)\right|_{\varsigma \in(-1,1)}
\end{aligned}
$$


3.2.2. Modified FGM type-II Let $A(v)$ and $C(\mathfrak{a})$ be two functional form satisfying all the conditions stated earlier where $\left.A(v)^{\bullet}\right|_{\left(\varsigma_{1}>0\right)}=v^{\varsigma_{1}}(1-v)^{1-\varsigma_{1}}$ and $\left.C(\mathfrak{a})^{\bullet}\right|_{\left(\varsigma_{2}>0\right)}=\mathfrak{a}^{\varsigma_{2}}(1-\mathfrak{a})^{1-\varsigma_{2}}$. Then, the corresponding BPRR-FGM (Type-II) can be derived from $\mathfrak{C}_{\varsigma, \varsigma_{1}, \varsigma_{2}}(v, \mathfrak{a})=v \mathfrak{a}\left[1+\varsigma A(v)^{\bullet} C(\mathfrak{a})^{\bullet}\right]$. Thus

$$
\begin{aligned}
\mathfrak{C}_{\varsigma, \varsigma_{1}, \varsigma_{2}}\left(z_{1}, z_{2}\right)= & \zeta_{\bullet 1}^{-1}\left[1-\exp \left(-\zeta_{1} \varrho_{\mathbf{P}}\left(z_{1}\right)\right)\right] \\
& \times \zeta_{\bullet 2}^{-1}\left[1-\exp \left(-\zeta_{2} \varrho_{\underline{\mathbf{P}}}\left(z_{2}\right)\right)\right] \\
& \left.\times\left[\begin{array}{c}
\left\{\zeta_{\bullet 1}^{-1}\left[1-\exp \left(-\zeta_{1} \varrho_{\mathbf{P}}\left(z_{1}\right)\right)\right]\right\}^{\varsigma_{1}} \\
\times\left\{\zeta_{\bullet 2}^{-1}\left[1-\exp \left(-\zeta_{2} \varrho_{\mathbf{P}}\left(z_{2}\right)\right)\right]\right\}^{\varsigma_{2}} \\
\times\left(1-\zeta_{\bullet 1}^{-1}\left[1-\exp \left(-\zeta_{1} \varrho_{\mathbf{P}}\left(z_{1}\right)\right)\right]\right)^{1-\varsigma_{1}} \\
\times\left(1-\zeta_{\bullet 2}^{-1}\left[1-\exp \left(-\zeta_{2} \varrho_{\mathbf{P}}\left(z_{2}\right)\right)\right]\right)^{1-\varsigma_{2}}
\end{array}\right)\right]
\end{aligned}
$$

3.2.3. Modified FGM type-III Let $\overline{C^{\bullet}(v)}=v\left[\log \left(1+v^{\bullet}\right)\right]$ and $\overline{D^{\bullet}(\mathfrak{a})}=\mathfrak{a}\left[\log \left(1+\mathfrak{a}^{\bullet}\right)\right]$ for all $A(v)$ and $C(\mathfrak{a})$ which satisfy all the conditions stated earlier. In this case, one can also derive a closed form expression for the associated CDF of the B-PRR-FGM (Type-III) from $\mathfrak{C}_{\varsigma}(v, \mathfrak{a})=v \mathfrak{a}\left(1+\varsigma \overline{C^{\bullet}(v)} \overline{D^{\bullet}(\mathfrak{a})}\right)$. Then

$$
\begin{aligned}
\mathfrak{C}_{\varsigma}\left(z_{1}, z_{2}\right)= & \zeta_{\bullet 1}^{-1}\left[1-\exp \left(-\zeta_{1} \varrho_{\mathbf{P}}\left(z_{1}\right)\right)\right] \\
& \times \zeta_{\bullet 2}^{-1}\left[1-\exp \left(-\zeta_{2} \varrho_{\mathbf{P}}\left(z_{2}\right)\right)\right] \\
& \times\left[1+\varsigma\left(\begin{array}{c}
\zeta_{\bullet 1}^{-1}\left[1-\exp \left(-\zeta_{1} \varrho_{\underline{\mathbf{P}}}\left(z_{1}\right)\right)\right] \\
\times \zeta_{\bullet 2}^{-1}\left[1-\exp \left(-\zeta_{2} \varrho_{\mathbf{P}}\left(z_{2}\right)\right)\right] \\
\times\left[\log \left(2-\zeta_{\bullet 1}^{-1}\left[1-\exp \left(-\zeta_{1} \varrho_{\mathbf{P}}\left(z_{1}\right)\right)\right]\right)\right] \\
\times\left[\log \left(2-\zeta_{\bullet 2}^{-1}\left[1-\exp \left(-\zeta_{2} \varrho_{\mathbf{P}}\left(z_{2}\right)\right)\right]\right)\right]
\end{array}\right)\right] .
\end{aligned}
$$

\subsection{Clayton copula}

The Clayton copula can be considered as $\mathfrak{C}\left(\mathfrak{a}_{1}, \mathfrak{a}_{2}\right)=\left.\left[\left(1 / \mathfrak{a}_{1}\right)^{\varsigma}+\left(1 / \mathfrak{a}_{2}\right)^{\varsigma}-1\right]^{-\varsigma^{-1}}\right|_{\varsigma \in(0,+\infty)}$. Setting $\mathfrak{a}_{1}=F(v)$ and $\mathfrak{a}_{2}=F(x)$, the B-PRR type can be derived from $\mathfrak{C}\left(\mathfrak{a}_{1}, \mathfrak{a}_{2}\right)=\mathfrak{C}\left(F\left(\mathfrak{a}_{1}\right), F\left(\mathfrak{a}_{2}\right)\right)$. Then

$$
\mathfrak{C}\left(z_{1}, z_{2}\right)=\left.\left\{\begin{array}{c}
\left\{\zeta_{\bullet 1}^{-1}\left[1-\exp \left(-\zeta_{1} \varrho_{\underline{\mathbf{P}}}\left(z_{1}\right)\right)\right]\right\}^{-\varsigma} \\
+\left\{\zeta_{\bullet 2}^{-1}\left[1-\exp \left(-\zeta_{2} \varrho_{\mathbf{P}}\left(z_{2}\right)\right)\right]\right\}^{-\varsigma}-1
\end{array}\right\}^{-\varsigma^{-1}}\right|_{\varsigma \in(0,+\infty)}
$$

Similarly, the M-PRR can be derived from

$$
\mathfrak{C}\left(x_{l}\right)=\left(\sum_{l=1}^{d}\left\{\zeta_{\bullet i}^{-1}\left[1-\exp \left(-\zeta_{i} \varrho_{\underline{\mathbf{P}}}\left(z_{l}\right)\right)\right]\right\}^{-\varsigma}+1-d\right)^{-\varsigma^{-1}} .
$$

\subsection{Renyi's entropy copula}

Using the theorem of Pougaza and Djafari (2011) where $\mathfrak{C}(v, \mathfrak{a})=z_{2} v+z_{1} \mathfrak{a}-z_{1} z_{2}$, the associated B-PRR can be derived from

$$
\begin{aligned}
\mathfrak{C}\left(z_{1}, z_{2}\right)= & z_{2} \zeta_{\bullet 1}^{-1}\left[1-\exp \left(-\zeta_{1} \varrho_{\underline{\mathbf{P}}}\left(z_{1}\right)\right)\right] \\
& +z_{1} \zeta_{\bullet 2}^{-1}\left[1-\exp \left(-\zeta_{2} \varrho_{\mathbf{P}}\left(z_{2}\right)\right)\right]-z_{1} z_{2} .
\end{aligned}
$$

\subsection{Ali-Mikhail-Haq copula}

Under the stronger Lipschitz condition, the Archimedean Ali-Mikhail-Haq copula can expressed as

$$
\mathfrak{C}(v, \mathfrak{a})=\left.v \mathfrak{a}\left[1-\varsigma v^{\bullet} v^{\bullet}\right]^{-1}\right|_{\tau \in(-1,1)},
$$


then for any $F_{\underline{\Phi}_{1}}\left(z_{1}\right)=1-\left.v^{\bullet}\right|_{[v \bullet=(1-v) \in(0,1)]}$ and $F_{\underline{\Phi}_{2}}\left(z_{2}\right)=1-\left.\mathfrak{a}^{\bullet}\right|_{[\mathfrak{a} \bullet=(1-\mathfrak{a}) \in(0,1)]}$ we have

$$
\mathfrak{C}\left(z_{1}, z_{2}\right)=\frac{\left\{\begin{array}{c}
\zeta_{\bullet 1}^{-1}\left[1-\exp \left(-\zeta_{1} \varrho_{\mathbf{P}}\left(z_{1}\right)\right)\right] \\
\times \zeta_{\bullet 2}^{-1}\left[1-\exp \left(-\zeta_{2} \varrho_{\mathbf{P}}\left(z_{2}\right)\right)\right]
\end{array}\right\}}{\left.\left.\left.1-\varsigma\left(\begin{array}{c}
\left\{1-\zeta_{\bullet 1}^{-1}\left[1-\exp \left(-\zeta_{1} \varrho_{\mathbf{P}}\left(z_{1}\right)\right)\right]\right\} \\
\times\left\{1-\zeta_{\bullet 2}^{-1}\left[1-\exp \left(-\zeta_{2} \varrho_{\mathbf{P}}\right.\right.\right.
\end{array} z_{2}\right)\right)\right]\right\}} .
$$

\section{Special PRR-G submodels}

In this Section we will provide many new distributions based on some common base line models namely: Loglogistic (LL), Weibull (W), Fréchet (F), Exponential (E), reciprocal exponential (RE), Lomax (Lx), reciprocal Lomax (RLx), Rayleigh (R), reciprocal Rayleigh (RR), Burr XII (BXII), Half-logistic (HL), Standard Gumbel (Gu), Lindley (L), Nadarajah-Haghighi (NH), Dagum (D), inverse flexible Weibull (IFW), Gumbel (Gu), Gompertz (Gz) and reciprocal Gompertz (RGz) (see Table 1). A special attention is given to the Poisson reciprocal Rayleigh exponential (PRRE) and the Poisson reciprocal Rayleigh Fréchet (PRRF) distributions. Figure 1 gives some plots of the PDF of the PRRE model. Figure 2 provides different plots of the HRF of the PRRE model. Figure 3 gives some plots of the PDF of the PRRF model. Figure 4 provides different plots of the HRF of the PRRF model. Based on Figure 1, the new density of the PRRE model can be "asymmetric right skewed with one peak and a heavy tail", "symmetric" and "left skewed with one peak". Based on Figure 2, the new hazard rate function (HRF) of the can be PRRE model "upside-down-constant", "increasing" and "decreasing-constant". Based on Figure 3, the new density of the PRRF model can be "asymmetric right skewed with one peak and a heavy tail", "symmetric" and "left skewed with one peak". Based on Figure 4, the new hazard rate function (HRF) of the can be PRRF model "upside-down", "increasing" and "decreasing". Based on Figure 1 and Figure 3, the family may be useful in modeling the "asymmetric right skewed with one peak and a heavy tail" real data sets, "symmetric" real data setsand "left skewed with one peak" real data sets.Based on Figure 3 and Figure 4, the family may be useful in modeling the real data sets which have "upside-down HRF", "upside-down-constant HRF", "increasing HRF", "decreasing HRF" and "decreasing-constant HRF".
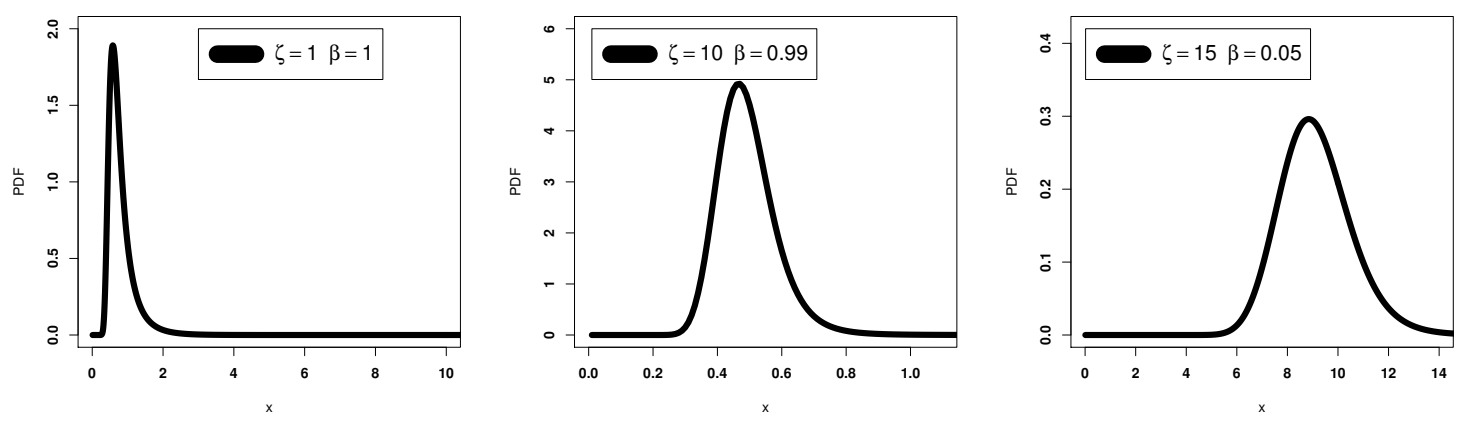

Figure 1. Plots of the PDF of the PRRE model. 

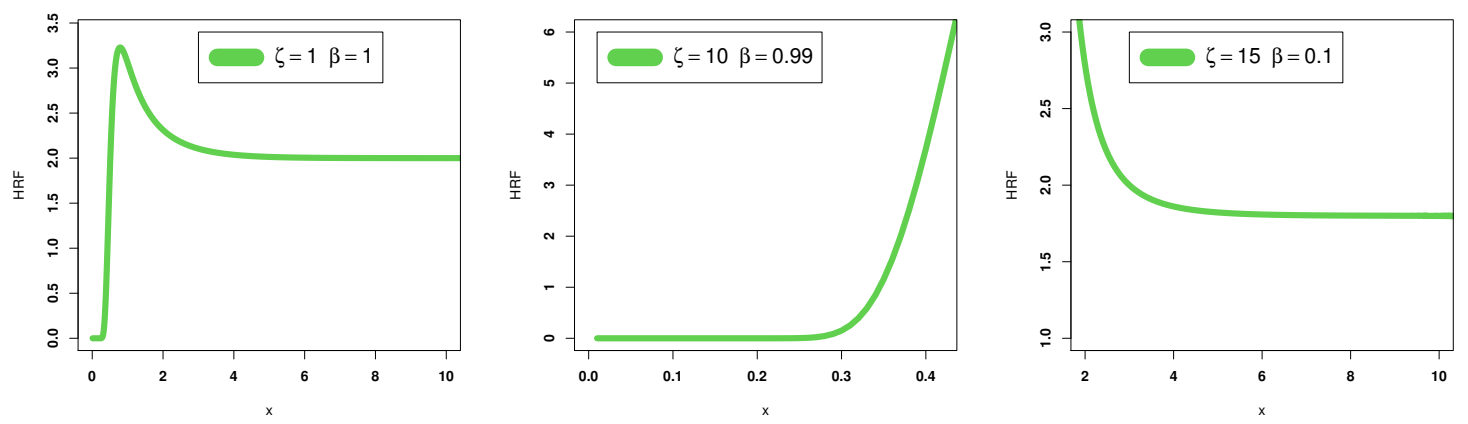

Figure 2. Plots of the HRF of the PRRE model.

Table 1: New submodels based on the new PRR-G family.

\begin{tabular}{llll}
\hline \hline No. & Base line model & $\nabla_{x, \underline{\mathbf{P}}}$ & Submodel \\
\hline \hline 1 & LL & $\left.\frac{\alpha}{\beta} x^{\alpha}\right|_{\beta, \alpha>0}$ & PRRLL \\
2 & $\mathrm{~W}$ & $\exp \left(x^{\beta}\right)-\left.1\right|_{\beta>0}$ & PRRW \\
3 & $\mathrm{~F}$ & {$\left.\left[\exp \left(x^{-\beta}\right)-1\right]^{-1}\right|_{\beta>0}$} & PRRF \\
4 & $\mathrm{E}$ & $\exp (\beta x)-\left.1\right|_{\beta>0}$ & PRRE \\
5 & $\mathrm{RE}$ & $\left.(1+x)^{\alpha}-\left.1\right|_{\alpha>0}\right]\left.^{-1}\right|_{\alpha>0}$ & PRRIE \\
6 & Lx & {$\left[\left(1+x^{-1}\right)^{\beta}-1\right]^{-1}$} & PRRLx \\
7 & RLx & $\exp (\alpha x)^{2}-\left.1\right|_{\alpha>0}$ & PRRRLx \\
8 & $\mathrm{R}$ & {$\left.\left[\exp \left(\alpha x^{-2}\right)-1\right]^{-1}\right|_{\alpha>0}$} & PRRR \\
9 & RR & $\left(1+x^{\beta}\right)^{\alpha}-\left.1\right|_{\beta, \alpha>0}$ & PRRRR \\
10 & BXII & $\left\{\left[\frac{1-\exp (-x)}{1+\exp (-x)}\right]^{-1}-1\right\}^{-1}$ & PRRBXII \\
11 & HL & $(\exp \{\exp [-(x)]\}-1)^{-1}$ & PRRHL \\
12 & Gu & $\exp (\alpha x)\left[\frac{1+\alpha+\alpha x}{1+\alpha}\right]^{-1}-\left.1\right|_{\alpha>0}$ & PRRGu \\
13 & L & $\exp \left[(1+\alpha x)^{\beta}-1\right]-\left.1\right|_{\alpha, \beta>0}$ & PRRL \\
14 & NH & $\left.\left\{\left[\frac{1-\exp (-\alpha x)}{1+\exp (-\alpha x)}\right]^{-1}-1\right\}^{-1}\right|_{\alpha>0}$ & PRRNH \\
15 & HL & $\left.\left\{\left[1+\left(\frac{x}{\lambda}\right)^{-\beta}\right]^{\alpha}-1\right\}^{-1}\right|_{\alpha, \beta, \lambda>0}$ & PRRHL \\
16 & Da & $\left.\left\{\exp \left[\exp \left(\frac{\alpha}{x}-\beta x\right)\right]-1\right\}^{-1}\right|_{\alpha>0}$ & PRRDa \\
17 & RFW & $\exp \{\beta[\exp (\alpha x)-1]\}-\left.1\right|_{\alpha>0}$ & PRRRFW \\
18 & Gz & $\left(\exp \left\{-\frac{\beta}{\alpha}\left[\exp \left(\frac{x}{\alpha}\right)-1\right]\right\}-1\right)$ & PRRz \\
19 & RGz & & PRRRGz \\
\hline \hline & &
\end{tabular}



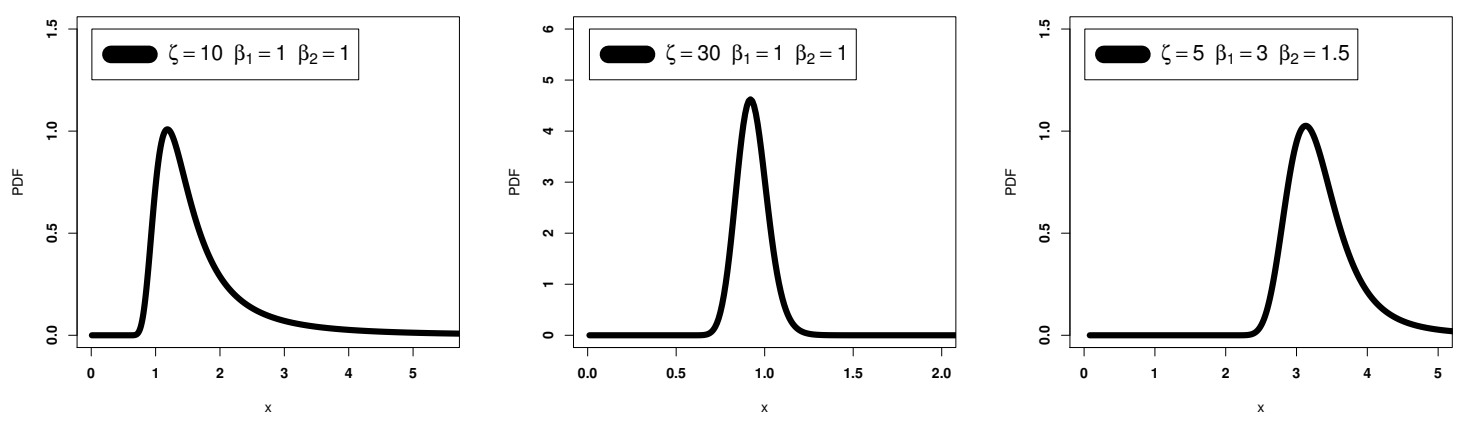

Figure 3. Plots of the PDF of the PRRF model.
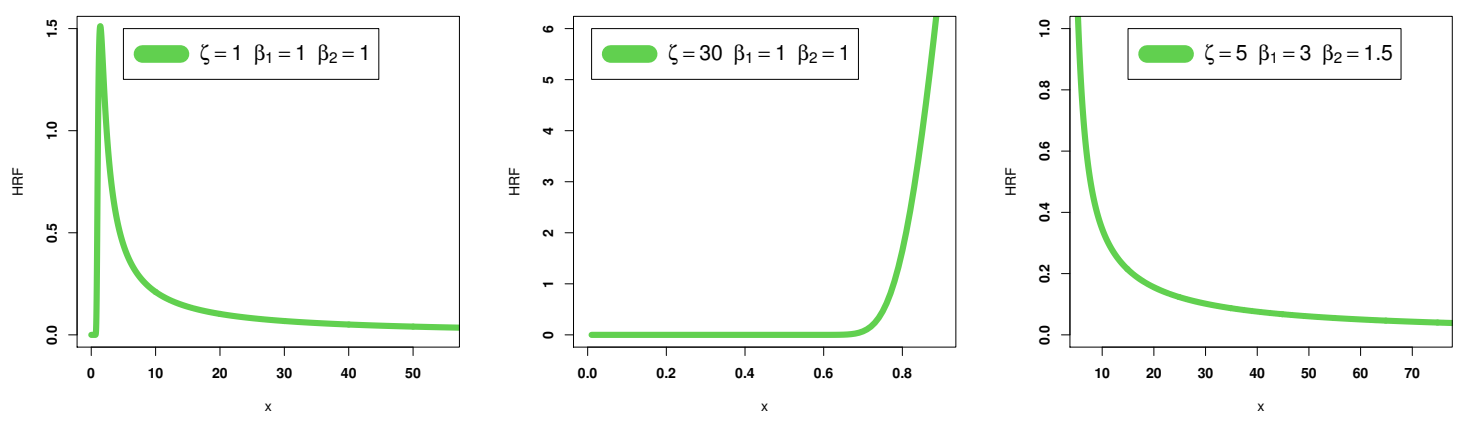

Figure 4. Plots of the HRF of the PRRF model.

\section{Parameter Estimation}

Here, we will consider the estimation of the unknown parameters $(\zeta, \underline{\mathbf{P}})$ of the new $\mathrm{G}$ family from complete samples by maximum likelihood method. Let $z_{1}, \cdots, x_{n}$ be a random sample (rs) from the PRR-G models parameter vector $\underline{\boldsymbol{\Omega}}=\left(\zeta, \underline{\mathbf{P}}^{\boldsymbol{\top}}\right)^{\top}$. The log-likelihood function for $\underline{\boldsymbol{\Omega}}$ is given by

$$
\begin{aligned}
\ell_{n}(\underline{\boldsymbol{\Omega}})= & n \log 2+n \log \zeta-n \log \zeta_{\bullet}+\sum_{i=1}^{n} \log g_{\underline{\mathbf{P}}}\left(x_{i}\right)+\sum_{i=1}^{n} \log \bar{G}_{\underline{\mathbf{P}}}\left(x_{i}\right) \\
& -\sum_{i=1}^{n} \frac{1}{\nabla_{x_{i}, \underline{\mathbf{P}}}^{2}}-3 \sum_{i=1}^{n} \log G_{\underline{\mathbf{P}}}\left(x_{i}\right)-\zeta \sum_{i=1}^{n} \exp \left(-\frac{1}{\nabla_{x_{i}, \underline{\mathbf{P}}}^{2}}\right) .
\end{aligned}
$$

The above log-likelihood function can be maximized numerically by using R (optim), SAS (PROC NLMIXED) or Ox program (sub-routine MaxBFGS), among others. For confidence interval (C.I.) estimation of the parameters, the elements of the observed information matrix $J(\underline{\boldsymbol{\Omega}})$ can be evaluated numerically, where

$$
U_{\zeta}=\frac{\partial}{\partial \zeta} \ell_{n}(\underline{\boldsymbol{\Omega}})=\frac{n}{\zeta}-\frac{n \exp (-\zeta)}{\zeta \bullet}-\sum_{i=1}^{n}\left\{1-\left[1-\exp \left(-\frac{1}{\nabla_{x_{i}, \underline{\mathbf{P}}}^{2}}\right)\right]^{\beta}\right\},
$$


and

$$
\begin{aligned}
U_{\underline{\mathbf{P}}_{\mathrm{P}}}= & \frac{\partial}{\partial \underline{\mathbf{P}}_{\mathbf{P}}} \ell_{n}(\underline{\boldsymbol{\Omega}})=\sum_{i=1}^{n} \frac{g_{\underline{\mathbf{P}}}^{\prime}\left(x_{i}\right)}{g_{\underline{\mathbf{P}}}\left(x_{i}\right)}-\sum_{i=1}^{n} \frac{G_{\underline{\mathbf{P}}}^{\prime}\left(x_{i}\right)}{\bar{G}_{\underline{\mathbf{P}}}\left(x_{i}\right)}+2 \sum_{i=1}^{n} q\left(x_{i}\right) \\
& +2(\beta-1) \sum_{i=1}^{n} \frac{q\left(x_{i}\right) \exp \left(-\frac{1}{\nabla_{x_{i}, \underline{\mathbf{P}}}^{2}}\right)}{1-\exp \left(-\frac{1}{\nabla_{x_{i}, \underline{\mathbf{P}}}^{2}}\right)}-3 \frac{G_{\underline{\mathbf{P}}}^{\prime}\left(x_{i}\right)}{G_{\underline{\mathbf{P}}}\left(x_{i}\right)} \\
& +2 \zeta \beta \sum_{i=1}^{n} \frac{q\left(x_{i}\right) \exp \left(-\frac{1}{\nabla_{x_{i}, \underline{\mathbf{P}}}^{2}}\right)}{\left[1-\exp \left(-\frac{1}{\nabla_{x_{i}, \underline{\mathbf{P}}}^{2}}\right)\right]^{1-\beta}} .
\end{aligned}
$$

where $\mathrm{P}$ is the number of parameters of the base line model,

$$
\begin{aligned}
g_{\underline{\mathbf{P}}}^{\prime}\left(x_{i}\right) & =\frac{\partial}{\partial \underline{\mathbf{P}}_{\mathbf{P}}} g_{\underline{\mathbf{P}}}\left(x_{i}\right), \\
G_{\underline{\mathbf{P}}}^{\prime} & \left(x_{i}\right)=\frac{\partial}{\partial \underline{\mathbf{P}}_{\mathbf{P}}} G_{\underline{\mathbf{P}}}\left(x_{i}\right),
\end{aligned}
$$

and

$$
q\left(x_{i}\right)=\nabla_{x, \underline{\mathbf{P}}}^{-3} \frac{G_{\underline{\mathbf{P}}}^{\prime}\left(x_{i}\right)}{\left[\bar{G}_{\underline{\mathbf{P}}}\left(x_{i}\right)\right]^{2}}
$$

Setting the nonlinear system of equations $U_{\zeta}=U_{\beta}=U_{\mathbf{P}_{\mathrm{P}}}=0$ and solving them simultaneously yields the maximum likelihood estimations (MLEs) of $\underline{\Omega}=\left(\zeta, \underline{\mathbf{P}}^{\top}\right)^{\bar{T}^{\mathrm{P}}}$. These equations can be solved numerically using convenient iterative method such as the Newton-Raphson type algorithms. For interval estimation of these parameters, we can obtain the observed information matrix $\mathbf{J}(\underline{\underline{\boldsymbol{\Omega}}})=\frac{\partial^{2} \ell_{n}(\underline{\boldsymbol{\Omega}})}{\partial m \partial n}$ (for $\left.m, n=\zeta, \mathbf{V}\right)$ which can be computed numerically. Under standard regularity conditions when $n \rightarrow+\infty$, the distribution of $(\underline{\widehat{\Omega}})$ can be approximated by a multivariate normal $N_{(\mathrm{P}+1)}\left(0, \mathbf{J}(\underline{\widehat{\Omega}})^{-1}\right)$ distribution to construct approximate confidence intervals for the parameters. Here, $\mathbf{J}(\underline{\widehat{\Omega}})$ is the total observed information matrix evaluated at $(\underline{\widehat{\Omega}})$. Large sample theory for these estimators delivers simple approximations that work well in finite samples. The normal approximation for the MLEs is easily handled numerically. Likelihood ratio tests can be performed for the proposed family in the usual way.

\section{Real data applications}

In this Section we analyze two real data sets. For data the first data set (the first application), we considered the standard one-parameter exponential distribution as our base line model and then we compare the fits of the PRRE distribution with other competitive exponential extensions such as Marshall-Olkin exponential (MOE) (Ghitany et al. (2005)), the odd Lindley exponential (OLE) (Almamy et al. (2018)), Moment exponential (ME) (Dara and Ahmad (2012)), generalized Marshall-Olkin exponential (GMOE) (Chakraborty and Handique (2017)), the Burr-Hatke exponential (BHE) (Yousof et al. (2018b)), beta exponential (BE) (Lee et al. (2007)), Kumaraswamy exponential (KE) (Cordeiro et al. (2010)), the Burr X exponential (BXE) distribution (Yousof et al. (2017b), Kumaraswamy Marshall-Olkin exponential (KMOE) (George and Thobias (2019)), Marshall-Olkin Kumaraswamy exponential (MOKE) (Chakraborty and Handique (2017)) and standard exponential (E) model. However, in the literature there are many other useful versions of the exponential distribution which can be used in comparison such as, Poisson-exponential (PE) distribution (Cancho et al. (2011)), transmuted exponentiated generalized 
exponential (TEGE) distribution (Yousof et al. (2017a)), Burr type XII exponential (BXIIE) distribution (Cordeiro et al. (2018)), The Burr X exponentiated exponential (BXEE) model (Khalil et al. (2019)), quasi Poisson Burr X exponentiated exponential (QPBXEE) distribution (Mansour et al. (2020b)), generalized odd log-logistic exponentiated exponential (GOLLEE) distribution (Mansour et al. (2020b)) and Mansour et al. (2020c)), among others.

For data set (the second application), we considered the standard two-parameters Fréchet distribution as our base line model and then we compare the fits of the PRRF distribution with other competitive Fréchet extensions such as the standard Fréchet (F) distribution, Marshal-Olkin Fréchet (MOF) distribution, Generalized MarshalOlkin Fréchet (GMOF) distribution, Kumaraswamy Fréchet (KF) distribution, beta Fréchet (BF) distribution, Kumaraswamy Marshal-Olkin Fréchet (KMOF) distribution and Marshal-Olkin Kumaraswamy Fréchet (MOKF) distribution. Some details related to the these copetitive model are available in Aboraya (2018), Aboraya (2019a,b) and Ibrahim et al. (2020).

For comparing models, we consider the Cramér-Von Mises (CM) and the Anderson-Darling (AD) and the Kolmogorov-Smirnov (KS) statistic (and its corresponding P-value), the Akaike Information Criterion $\left(\mathrm{C}_{1}\right)$, Bayesian Information Criterion $\left(\mathrm{C}_{2}\right)$, consistent Akaike Information Criterion $\left(\mathrm{C}_{3}\right)$ and Hannan-Quinn Information Criterion $\left(\mathrm{C}_{4}\right)$ where

$$
\begin{gathered}
\mathrm{CM}=\left(1+\frac{1}{2 n}\right)\left[\frac{1}{12 n}+\sum_{h=1}^{n}\left(z_{h}-\frac{2 h-1}{2 n}\right)^{2}\right], \\
\mathrm{AD}=a_{(n)}\left(n+\frac{1}{n} \sum_{l=1}^{n} q_{l}\right), \\
\mathrm{C}_{1}=-2 \widehat{\ell}+2 \mathrm{P} \\
\mathrm{C}_{2}=-2 \widehat{\ell}+\mathrm{P} \log (n) \\
\mathrm{C}_{3}=-2 \widehat{\ell}+\frac{2 n \mathrm{P}}{n-\mathrm{P}-1}, \\
\mathrm{C}_{4}=-2 \widehat{\ell}+2 \mathrm{P} \log [\log (n)],
\end{gathered}
$$

where

$$
a_{(n)}=1+\frac{9}{4} n^{-2}+\frac{3}{4} n^{-1}
$$

and

$$
q_{l}=(2 l-1) \log \left[z_{l}\left(1-z_{n-l+1}\right)\right],
$$

where $z_{l}=F\left(y_{l}\right)$, the $y_{l}$ 's values are the ordered observations and $n$ is the sample size. However, other potential goodness-of-fit statistic tests for validation such as Nikulin-Rao-Robson statistic test and the modified NikulinRao-Robson statistic test may be used (see Goual et al. (2019), Abouelmagd et al. (2019), Ibrahim et al. (2019 and 2020), Goual et al. (2020), Goual and Yousof (2020), Yadav et al. (2020) and Yousof et al. (2021)).

\subsection{First application (failure times data)}

The failure times data set is given in Gross and Clark (1975)). The data represents the lifetime data relating to relief times (in minutes) of patients receiving an analgesic. This data was recently analyzed by Al-babtain et al. (2020) and Ibrahim et al. (2020). Table 2 lists the MLEs, standard errors (SEs) and 95\% C.I.s (LC.I., UC.I.). Table 3 lists the $\mathrm{C}_{1}$, $\mathrm{C}_{2}, \mathrm{C}_{3}, \mathrm{C}_{4}, \mathrm{AD}, \mathrm{CM}, \mathrm{K} . \mathrm{S}$. and its p-value. Figure 3 gives the total time in test (TTT) plot (see Aarset (1987)) for the relief times data along with the corresponding quantile-quantile (QQ) plot, box plot and the nonparametric Kernel density estimation (N-KDE) plot. Based on Figure 5, the HRF of the relief times is "monotonically increasing HRF" (top right plot) and this data has an extreme value ( see top right and bottom right plots) and its density is right skewed and bimodal. Figure 6 gives the estimated PDF (E-PDF), estimated CDF (E-CDF), estimated HRF (E-HRF) and P-P plot for relief times data. Based on results of Table 3 and Table 4, it is concluded that the PRRE model is much better than the exponential, odd Lindley exponential, Marshall-Olkin exponential, moment exponential, the 
logarithmic Burr-Hatke exponential, generalized Marshall-Olkin exponential, Beta exponential, Marshall-Olkin Kumaraswamy exponential, Kumaraswamy exponential, the Burr X exponential and Kumaraswamy MarshallOlkin exponential models with $\mathrm{C}_{1}=34.86, \mathrm{C}_{2}=36.85, \mathrm{C}_{3}=35.56, \mathrm{C}_{4}=35.24, \mathrm{AD}=0.146, \mathrm{CM}=0.025, \mathrm{~K} . \mathrm{S}=0.092$ and p-value $=0.9955$ so the new lifetime model is a good alternative to these models in modeling relief times data set. According to Figures 6, the PRRE distribution provides adequate fits to the empirical functions.

Table 2: MLEs, SEs, C.I.s (in parentheses) values for the relief times data.

\begin{tabular}{|c|c|c|}
\hline Models & & Estimates \\
\hline$\overline{\mathrm{E}(\beta)}$ & $\begin{array}{c}\text { MLE } \\
\text { SE } \\
\text { (LC.I., UC.I.) }\end{array}$ & $\begin{array}{c}0.526 \\
(0.117) \\
(0.29,0.75)\end{array}$ \\
\hline $\operatorname{OLE}(\beta)$ & $\begin{array}{c}\text { MLE } \\
\text { SE } \\
\text { (LC.I., UC.I.) }\end{array}$ & $\begin{array}{c}0.6044 \\
(0.0535) \\
(0.5,0.7)\end{array}$ \\
\hline $\operatorname{ME}(\beta)$ & $\begin{array}{c}\text { MLE } \\
\text { SE } \\
\text { (LC.I., UC.I.) }\end{array}$ & $\begin{array}{c}0.950 \\
(0.150) \\
(0.66,1.24)\end{array}$ \\
\hline $\operatorname{BHE}(\beta)$ & $\begin{array}{c}\text { MLE } \\
\text { SE } \\
\text { (LC.I., UC.I.) }\end{array}$ & $\begin{array}{c}0.5263 \\
(0.118) \\
(0.43,0.63)\end{array}$ \\
\hline $\operatorname{MOE}(\zeta, \beta)$ & $\begin{array}{c}\text { MLE } \\
\text { SE } \\
\text { (LC.I., UC.I.) }\end{array}$ & $\begin{array}{c}54.474,2.316 \\
(35.582),(0.374) \\
(0,124.21),(1.58,3.04)\end{array}$ \\
\hline $\operatorname{GMOE}(\alpha, \zeta, \beta)$ & $\begin{array}{c}\text { MLE } \\
\text { SE } \\
\text { (LC.I., UC.I.) }\end{array}$ & $\begin{array}{c}0.519,89.462,3.169 \\
(0.256),(66.278),(0.772) \\
(0.02,1.02),(0,219.37),(1.66,4.68)\end{array}$ \\
\hline $\mathrm{KE}(\alpha, \zeta, \beta)$ & $\begin{array}{c}\text { MLE } \\
\text { SE } \\
\text { (LC.I., UC.I.) }\end{array}$ & $\begin{array}{c}83.756,0.568,3.330 \\
(42.361),(0.326),(1.188) \\
(0.73,166.78),(0,1.21),(1.00,5.66)\end{array}$ \\
\hline $\mathrm{BE}(\alpha, \zeta, \beta)$ & $\begin{array}{c}\text { MLE } \\
\text { SE } \\
\text { (LC.I., UC.I.) }\end{array}$ & $\begin{array}{c}81.633,0.542,3.514 \\
(120.41),(0.327),(1.410) \\
(0,317.63),(0,1.18),(0.75,6.28)\end{array}$ \\
\hline $\operatorname{MOKE}(\alpha, a, \zeta, \beta)$ & $\begin{array}{c}\text { MLE } \\
\text { SE } \\
\text { (LC.I., UC.I.) }\end{array}$ & $\begin{array}{c}0.133,33.232,0.571,1.669 \\
(0.332),(57.837),(0.721),(1.814) \\
(0,0.78),(0,146.59),(0,1.98),(0,5.22)\end{array}$ \\
\hline $\operatorname{KwMOE}(\alpha, a, \zeta, \beta)$ & $\begin{array}{c}\text { MLE } \\
\text { SE } \\
\text { (LC.I., UC.I.) }\end{array}$ & $\begin{array}{c}8.868,34.826,0.299,4.899 \\
(9.146),(22.312),(0.239),(3.176) \\
(10.9,46.8),(0,78.6),(0,0.8),(0,11.12)\end{array}$ \\
\hline $\operatorname{BXE}(\zeta, \beta)$ & $\begin{array}{c}\text { MLE } \\
\text { SE } \\
\text { (LC.I., UC.I.) }\end{array}$ & $\begin{array}{c}1.1635,0.3207 \\
(0.33),(0.03) \\
(0.5,1.8),(0.26,0.4)\end{array}$ \\
\hline $\mathbf{E I B X E}(\zeta, \beta)$ & $\begin{array}{c}\text { MLE } \\
\text { SE } \\
\text { (LC.I., UC.I.) }\end{array}$ & $\begin{array}{c}3.0215,0.3416 \\
(1.558),(0.0336) \\
(0,6),(0,0.94)\end{array}$ \\
\hline
\end{tabular}



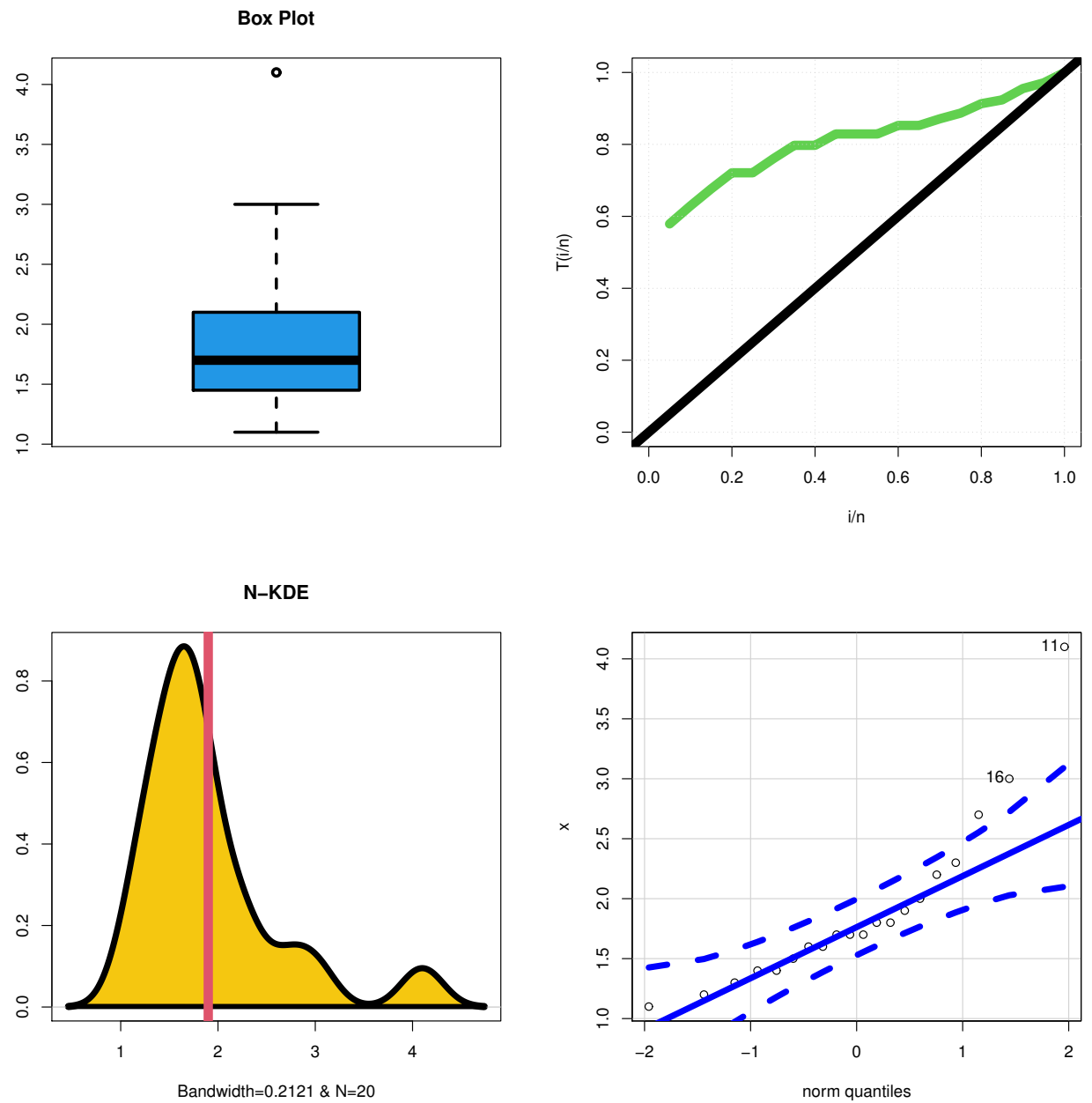

Figure 5. Box, TTT, N-KDE, QQ plots for the relief times data.

Table 3: $\mathrm{C}_{1}, \mathrm{C}_{2}, \mathrm{C}_{3}, \mathrm{C}_{4}$ for the relief times data.

\begin{tabular}{ccccc}
\hline \hline Models & $\mathrm{C}_{1}$ & $\mathrm{C}_{2}$ & $\mathrm{C}_{3}$, & $\mathrm{C}_{4}$ \\
\hline \hline $\mathrm{E}$ & 67.67 & 68.67 & 67.89 & 67.87 \\
$\mathrm{OLE}$ & 49.12 & 50.11 & 49.32 & 49.30 \\
$\mathrm{ME}$ & 54.32 & 55.31 & 54.54 & 54.50 \\
$\mathrm{BHE}$ & 67.67 & 68.67 & 67.89 & 67.87 \\
$\mathrm{MOE}$ & 43.51 & 45.51 & 44.22 & 43.90 \\
$\mathrm{GMOE}$ & 42.75 & 45.74 & 44.25 & 43.34 \\
$\mathrm{KE}$ & 41.78 & 44.75 & 43.28 & 42.32 \\
$\mathrm{BE}$ & 43.48 & 46.45 & 44.98 & 44.02 \\
MOKE & 41.58 & 45.54 & 44.25 & 42.30 \\
KMOE & 42.83 & 46.84 & 45.55 & 43.60 \\
$\mathrm{BXE}$ & 48.13 & 50.12 & 48.81 & 48.52 \\
PRRE & $\mathbf{3 4 . 8 5 6 2}$ & $\mathbf{3 6 . 8 5}$ & $\mathbf{3 5 . 5 6}$ & $\mathbf{3 5 . 2 4}$ \\
\hline \hline
\end{tabular}


Table 4: AD, CM, K.S. and p-value for the relief times data.

\begin{tabular}{ccccc}
\hline \hline Models & AD & CM & K.S. & p-value \\
\hline \hline E & 4.60 & 0.96 & 0.44 & 0.004 \\
OLE & 1.33 & 0.22 & 0.85 & $<0.001$ \\
ME & 2.76 & 0.53 & 0.32 & 0.07 \\
BHE & 0.62 & 0.105 & 0.44 & $<0.001$ \\
MOE & 0.84 & 0.14 & 0.18 & 0.55 \\
GMOE & 0.51 & 0.08 & 0.15 & 0.78 \\
KE & 0.45 & 0.07 & 0.14 & 0.86 \\
BE & 0.70 & 0.12 & 0.16 & 0.80 \\
MOKE & 0.60 & 0.11 & 0.14 & 0.87 \\
KMOE & 1.08 & 0.19 & 0.15 & 0.86 \\
BXE & 1.39 & 0.24 & 0.25 & 0.17 \\
PRRE & $\mathbf{0 . 1 4 6}$ & $\mathbf{0 . 0 2 5}$ & $\mathbf{0 . 0 9 2}$ & $\mathbf{0 . 9 9 5 5}$ \\
\hline \hline
\end{tabular}
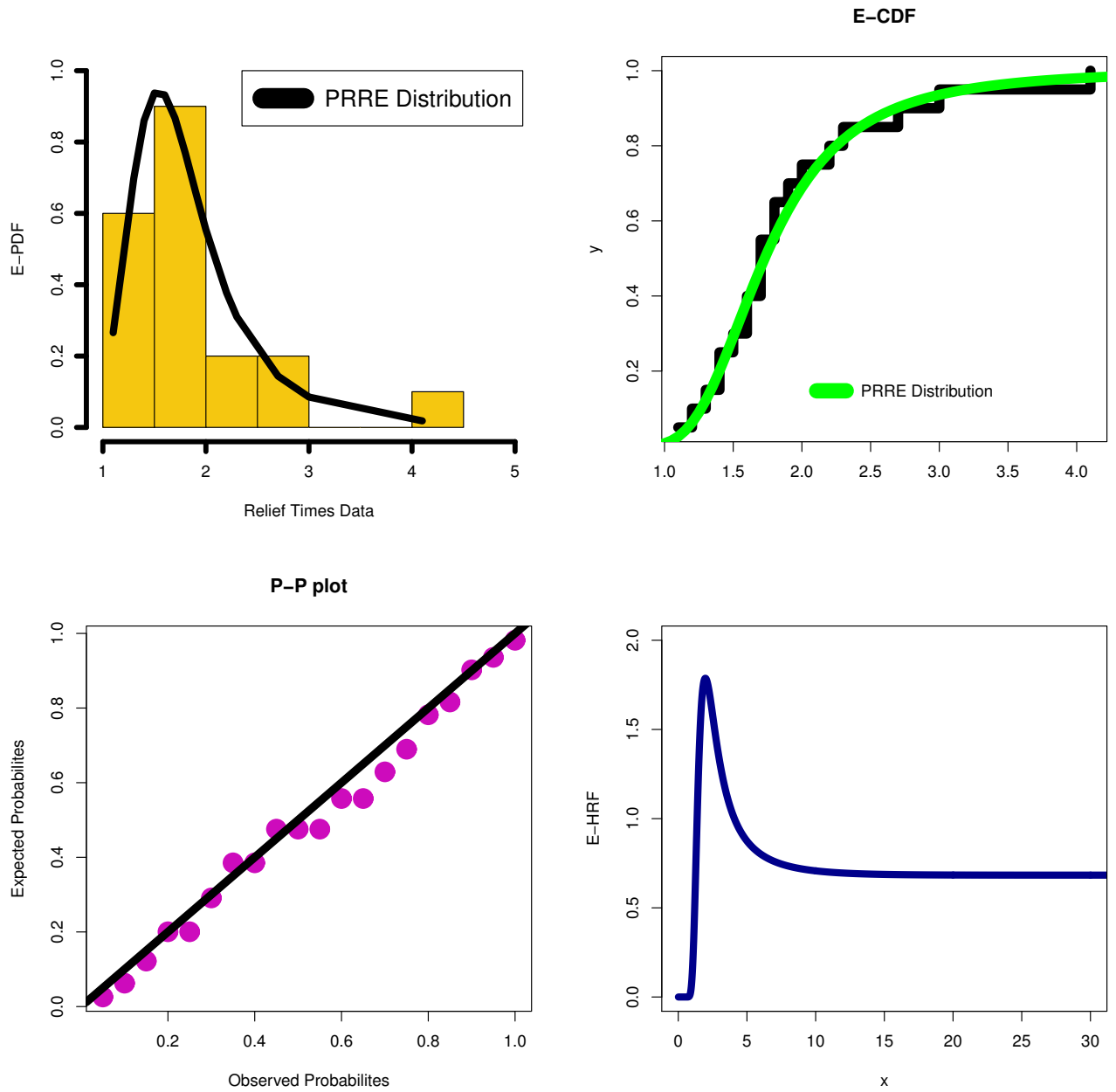

Figure 6. E-PDF, E-CDF, P-P and E-HRF for relief times data. 


\subsection{Second application (gauge lengths data)}

The fgauge lengths data set consists of 74 observations (see Kundu and Raqab (2009)). Table 5 lists the MLEs, SEs confidence intervals (C.I.s) for the gauge lengths data. Table 6 lists the $\mathrm{C}_{1}, \mathrm{C}_{2}, \mathrm{C}_{3}, \mathrm{C}_{4}, \mathrm{AD}, \mathrm{CM}, \mathrm{K} . \mathrm{S}$. and p-value. Figure 5 gives the total time test (TTT) plot (Aarset (1987)) for the relief times data along with the corresponding box plot, QQ plot and the N-KDE plot. Based on Figure 7, the HRF of the gauge lengths data is "monotonically increasing HRF" (see top right plot) and this data has no extreme observation (see top right and bottom right plots) and its density is semi-bimodal. Figure 6 gives the E-PDF, E-CDF, E-HRF and P-P plot for gauge lengths data. Based on results Table 5 and Table 7, it is concluded that the PRRF model is much better than the Fréchet, Marshal-Olkin Fréchet, Generalized Marshal-Olkin Fréchet, Kumaraswamy Fréchet, beta Fréchet, Kumaraswamy Marshal-Olkin Fréchet and Marshal-Olkin Kumaraswamy Fréchet models with $\mathrm{C}_{1}=109.36, \mathrm{C}_{2}=116.28, \mathrm{C}_{3}=109.70, \mathrm{C}_{4}=112.121, \mathrm{AD}=0.33, \mathrm{CM}=0.047, \mathrm{~K} . \mathrm{S}=0.060$ and $\mathrm{p}$-value $=0.9495$ so the new lifetime model is a good alternative to these models in modeling gauge lengths data set. According to Figures 6, the PRRF distribution provides adequate fits to the emPRRical functions.
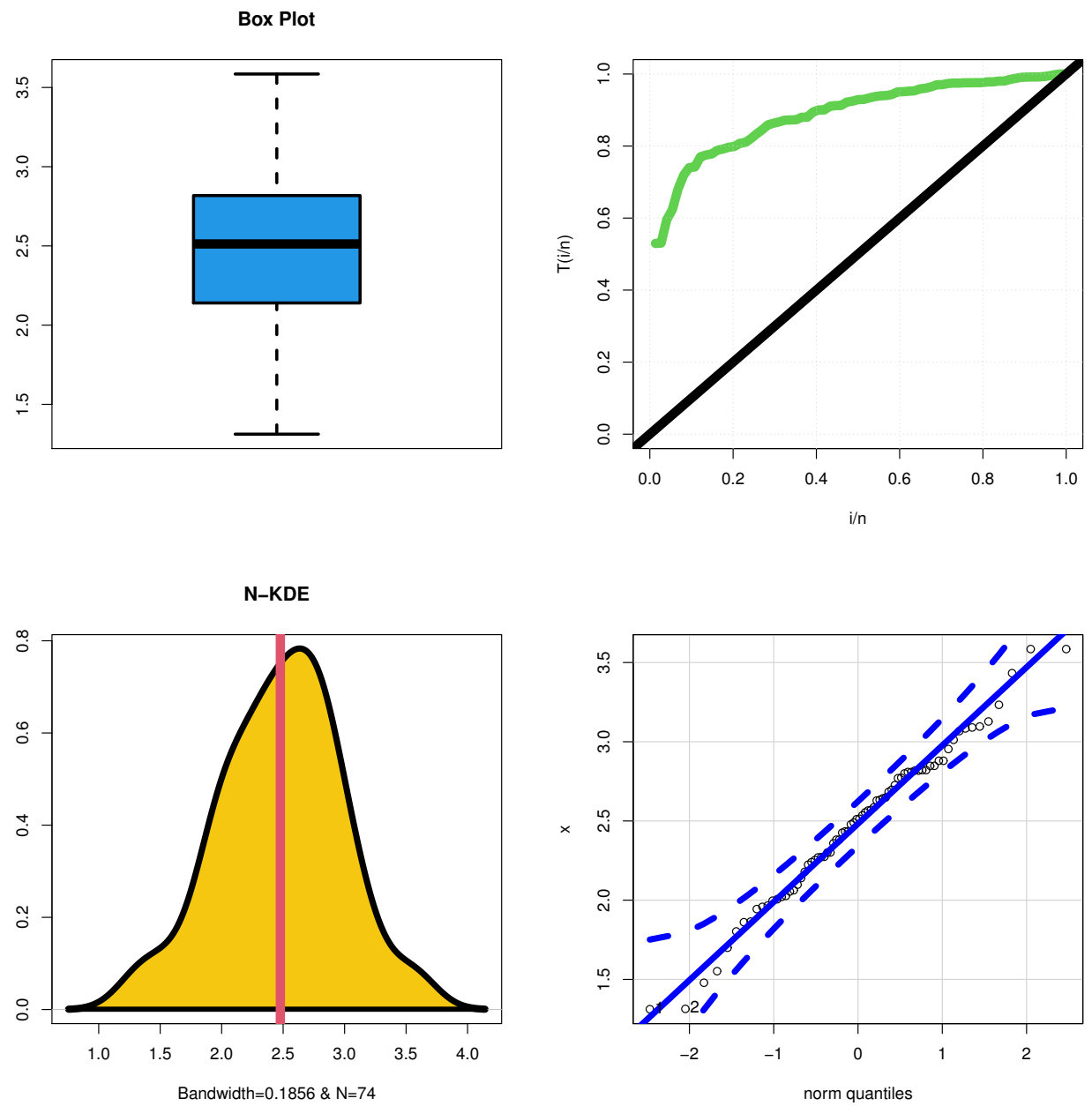

Figure 7. Box, TTT, N-KDE, QQ plots for the gauge lengths data. 
Table 5: MLEs, SEs, C.I.s (in parentheses) values for the gauge lengths data.

\begin{tabular}{|c|c|c|}
\hline Models & & Estimates \\
\hline$\overline{F\left(\beta_{1}, \beta_{2}\right)}$ & "MLE & $4.110,2.169$ \\
\hline \multirow{5}{*}{$\operatorname{MOF}\left(\alpha, \beta_{1}, \beta_{2}\right)$} & SE & $(0.323),(0.065)$ \\
\hline & (LC.I., UC.I.) & $(3.48,4.74),(2.04,2.29)$ \\
\hline & MLE & $80.338,8.0311 .419$ \\
\hline & SE & $(62.007),(0.764),(0.109)$ \\
\hline & (LC.I., UC.I.) & $(0,201.87),(6.53,9.53),(1.21,1.63)$ \\
\hline \multirow[t]{3}{*}{$\operatorname{GMOF}\left(\alpha, \zeta, \beta_{1}, \beta_{2}\right)$} & MLE & $3.702,63.707,5.918,1.577$ \\
\hline & SE & $(2.683),(38.655),(0.945),(0.139)$ \\
\hline & (LC.I., UC.I.) & $(0,8.96),(0,139.47),(4.06,7.77),(1.30,1.85)$ \\
\hline \multirow{3}{*}{$\mathrm{KF}\left(\alpha, \zeta, \beta_{1}, \beta_{2}\right)$} & MLE & $3.218,217.031,1.005,4.384$ \\
\hline & SE & $(1.036),(268.565),(0.223),(1.012)$ \\
\hline & (LC.I., UC.I.) & $(1.18,5.25),(0,743.42),(0.57,1.44),(2.40,6.37)$ \\
\hline \multirow[t]{3}{*}{$\mathrm{BF}\left(\alpha, \zeta, \beta_{1}, \beta_{2}\right)$} & MLE & $2.039,5.857,0.242,37.179$ \\
\hline & SE & $(1.015),(1.813),(0.377),(33.510)$ \\
\hline & (LC.I., UC.I.) & $(0.04,4.02),(2.30,9.41),(0,0.98),(0,102.85)$ \\
\hline \multirow{3}{*}{$\operatorname{KMOF}\left(\alpha, a, \zeta, \beta_{1}, \beta_{2}\right)$} & MLE & $0.016,0.827,16.985,0.894,25.127$ \\
\hline & SE & $(0.023),(0.789),(24.975),(0.396), 19.688)$ \\
\hline & (LC.I., UC.I.) & $(0,0.06),(0,2.37),(0,65.93),(0.11,1.67),(0,63.72)$ \\
\hline \multirow[t]{3}{*}{$\operatorname{MOKF}\left(\alpha, a, \zeta, \beta_{1}, \beta_{2}\right)$} & MLE & $7.995,2.933,35.707,1.221,2.415$ \\
\hline & SE & $(13.063),(0.825),(41.500),(0.412),(1.032)$ \\
\hline & (LC.I., UC.I.) & $(0,33.6),(1.31,4.6),(0,117.1),(0.41,2.03),(0.39,4.44)$ \\
\hline \multirow{3}{*}{$\operatorname{PRRF}\left(\zeta, \beta_{1}, \beta_{2}\right)$} & MLE & $994.5,8.3,0.222$ \\
\hline & SE & $(10.5),(0.55),(0.25)$ \\
\hline & (LC.I., UC.I.) & $(973,1015),(7.2,9.4),(0,0.72)$ \\
\hline
\end{tabular}

Table 6: $\mathrm{C}_{1}, \mathrm{C}_{2}, \mathrm{C}_{3}, \mathrm{C}_{4}$ for the gauge lengths data.

\begin{tabular}{ccccc}
\hline \hline Models & $\mathrm{C}_{1}$ & $\mathrm{C}_{2}$ & $\mathrm{C}_{3}$, & $\mathrm{C}_{4}$ \\
\hline \hline $\mathrm{F}$ & 142.02 & 146.63 & 142.19 & 143.86 \\
$\mathrm{MOF}$ & 115.06 & 121.96 & 115.40 & 117.81 \\
$\mathrm{GMOF}$ & 112.80 & 122.00 & 113.37 & 126.48 \\
$\mathrm{KF}$ & 113.68 & 122.82 & 114.25 & 117.36 \\
$\mathrm{BF}$ & 112.63 & 121.84 & 113.21 & 116.30 \\
$\mathrm{KMOF}$ & 113.30 & 124.82 & 114.18 & 117.90 \\
MOKF & 113.19 & 124.68 & 114.07 & 117.78 \\
PRRF & $\mathbf{1 0 9 . 3 6}$ & $\mathbf{1 1 6 . 2 8}$ & $\mathbf{1 0 9 . 7 0}$ & $\mathbf{1 1 2 . 1 2 1}$ \\
\hline \hline
\end{tabular}

Table 7: AD, CM, K.S. and p-value for the gauge lengths data.

\begin{tabular}{ccccc}
\hline \hline Models & AD & CM & K.S. & p-value \\
\hline \hline F & 2.93 & 0.46 & 0.151 & 0.09 \\
MOF & 0.78 & 0.12 & 0.072 & 0.86 \\
GMOF & 0.39 & 0.07 & 0.065 & 0.94 \\
KF & 0.37 & 0.07 & 0.065 & 0.92 \\
BF & 0.44 & 0.07 & 0.064 & 0.93 \\
KMOF & 0.36 & 0.055 & 0.066 & 0.95 \\
MOKF & 0.36 & 0.055 & 0.063 & 0.94 \\
PRRF & $\mathbf{0 . 3 3}$ & $\mathbf{0 . 0 4 7}$ & $\mathbf{0 . 0 6 0}$ & $\mathbf{0 . 9 4 9 5}$ \\
\hline \hline
\end{tabular}



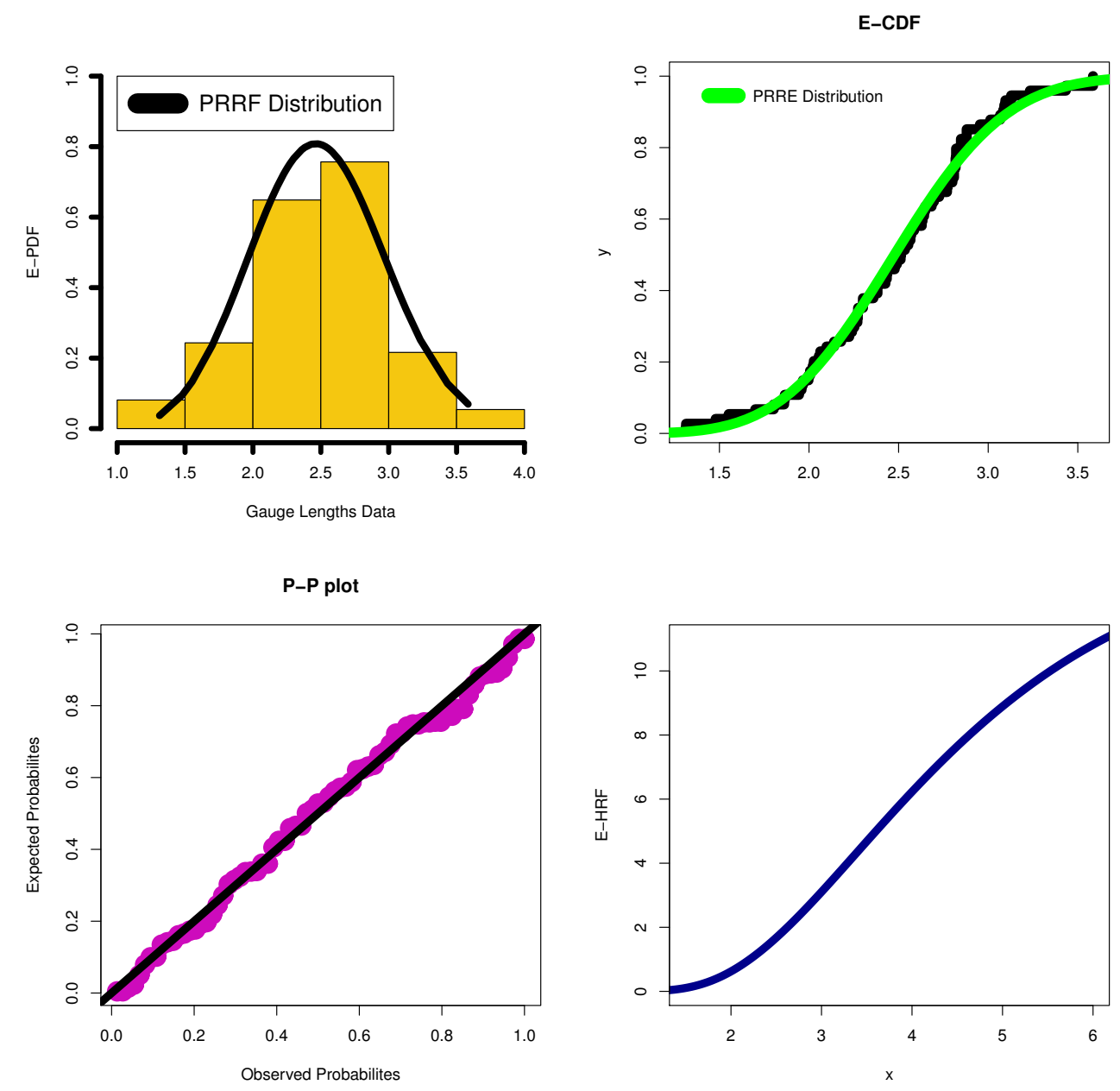

Figure 8. E-PDF, E-CDF, P-P and E-HRF plot for gauge lengths data.

\section{Concluding remarks}

In this work, a new one-parameter compound $\mathrm{G}$ family of continuous distributions is derived and studied. Relevant statistical properties such as moments, incomplete moments and moment generating function are derived. The density of the new family is re-expressed in terms of the exponentiated $G$ family. The new density can be "asymmetric right skewed with one peak and a heavy tail", "symmetric" and "left skewed with one peak". The corresponding hazard function can be "upside-down", "upside-down-constant", "increasing", "decreasing" and "decreasing-constant".

Many bivariate types have been also derived via different common copulas. The estimation of the model parameters is performed by the maximum likelihood method. The usefulness and flexibility of the new family is illustrated by means of two real data sets. The new family is better than the odd Lindley family, Marshall-Olkin family, the Burr-Hatke family, generalized Marshall-Olkin family, Beta family, Marshall-Olkin Kumaraswamy family, Kumaraswamy family, the Burr X family and Kumaraswamy Marshall-Olkin family in modeling the bimodal right skewed relief times data set with $\mathrm{C}_{1}=34.86, \mathrm{C}_{2}=36.85, \mathrm{C}_{3}=35.56, \mathrm{C}_{4}=35.24, \mathrm{AD}=0.146, \mathrm{CM}=0.025$, $\mathrm{K} . \mathrm{S}=0.092$ and $\mathrm{p}$-value $=0.9955$ so the new family could be considered as a good alternative to these families. 
The new family is better than the Marshal-Olkin family, Generalized Marshal-Olkin family, Kumaraswamy family, beta family, Kumaraswamy Marshal-Olkin family and Marshal-Olkin Kumaraswamy family in modeling the gauge lengths data set with $\mathrm{C}_{1}=109.36, \mathrm{C}_{2}=116.28, \mathrm{C}_{3}=109.70, \mathrm{C}_{4}=112.121, \mathrm{AD}=0.33, \mathrm{CM}=0.047, \mathrm{~K} . \mathrm{S}=0.060$ and $\mathrm{p}$ value $=0.9495$ so the new family could be considered as a good alternative to these families .

\section{REFERENCES}

1. Aarset, M. V. (1987). How to identify a bathtub hazard rate. IEEE Transactions on Reliability, 36(1), 106-108.

2. Aboraya, M. (2018). A new flexible lifetime model with statistical properties and applications. Pakistan Journal of Statistics and Operation Research, 881-901.

3. Aboraya, M. (2019a). The extended generalized inverse Weibull distribution and its applications, Journal of Statistics and Applications, 2(1), 1-17.

4. Aboraya, M. (2019b). A new extremely flexible version of the exponentiated Weibull model: theorem and applications to reliability and medical data sets. Pakistan Journal of Statistics and Operation Research, 195-215.

5. Abouelmagd, T. H. M., Hamed, M. S., Hamedani, G. G., Ali, M. M., Goual, H., Korkmaz, M. C. and Yousof, H. M. (2019). The zero truncated Poisson Burr X family of distributions with properties, characterizations, applications, and validation test. Journal of Nonlinear Sciences and Applications, 12(5), 314-336.

6. Abouelmagd, T. H. M., Hamed. M. S., Handique, L. Goual, H. Ali, M. M., Yousof, H. M. and Korkmaz, M. C. (2019). A new class of distributions based on the zero truncated Poisson distribution with properties and applications. Journal of Nonlinear Sciences and Applications, 12(3), 152-164.

7. Al-babtain, A. A., Elbatal, I. and Yousof, H. M. (2020). A New Flexible Three-Parameter Model: Properties, Clayton Copula, and Modeling Real Data. Symmetry, 12(3), 440.

8. Ali, M. M., Ibrahim, M. and Yousof, H. M. (2021a). Expanding the Burr X model: properties, copula, real data modeling and different methods of estimation. Optimal Decision Making in Operations Research \& Statistics: Methodologies and Applications, to appear.

9. Ali, M. M., Mikhail, N. N. and Haq, M. S. (1978). A class of bivariate distributions including the bivariate logistic. Journal of multivariate analysis, 8(3), 405-412.

10. Ali, M. M., Yousof, H. M. and Ibrahim, M. (2021b). A new version of the generalized Rayleigh distribution with copula, properties, applications and different methods of estimation. Optimal Decision Making in Operations Research \& Statistics: Methodologies and Applications, to appear.

11. Alizadeh, M., Yousof, H. M. Rasekhi, M. and Altun, E. (2019). The odd log-logistic Poisson-G Family of distributions, Journal of Mathematical Extensions, 12(3), 81-104.

12. Alizadeh, M., Jamal, F., Yousof, H. M., Khanahmadi, M. and Hamedani, G. G. (2020a). Flexible Weibull generated family of distributions: characterizations, mathematical properties and applications. University Politehnica of Bucharest Scientific BulletinSeries A-Applied Mathematics and Physics, 82(1), 145-150.

13. Alizadeh, M., Yousof, H. M., Jahanshahi, S. M. A., Najibi, S. M. and Hamedani, G. G. (2020b). The transmuted odd log-logistic-G family of distributions. Journal of Statistics and Management Systems, 23(4), 1-27.

14. Aryal, G. R. and Yousof, H. M. (2017). The exponentiated generalized-G Poisson family of distributions. Economic Quality Control, 32(1), 1-17.

15. Brito, E., Cordeiro, G. M., Yousof, H. M., Alizadeh, M. and Silva, G. O. (2017). Topp-Leone Odd Log-Logistic Family of Distributions, Journal of Statistical Computation and Simulation, 87(15), 3040-3058.

16. Cancho, V. G., Louzada-Neto, F. and Barriga, G. D. (2011). The Poisson-exponential lifetime distribution. Computational Statistics \& Data Analysis, 55(1), 677-686.

17. Chakraborty, S. and Handique, L. (2017). The generalized Marshall-Olkin-Kumaraswamy-G family of distributions. Journal of data Science, 15(3), 391-422.

18. Cordeiro, G.M., Ortega E.M.M., Nadarajah, S. (2010). The Kumaraswamy Weibull distribution with application to failure data. J. Frankl Inst, 347, 1399-1429.

19. Cordeiro, G. M., Yousof, H. M., Ramires, T. G. and Ortega, E. M. (2018). The Burr XII system of densities: properties, regression model and applications. Journal of Statistical Computation and Simulation, 88(3), 432-456.

20. Dara, S.T. and Ahmad, M. (2012). Recent Advances in Moment Distributions and their Hazard Rate. Ph.D. Thesis. National College of Business Administration and Economics, Lahore, Pakistan.

21. Eugene, N., Lee, C. and Famoye, F. (2002). Beta-normal distribution and its applications. Commun. Stat. Theory Methods, 31, 497512 .

22. Elgohari, H. and Yousof, H. M. (2020a). A Generalization of Lomax Distribution with Properties, Copula and Real Data Applications Pakistan Journal of Statistics and Operation Research, 16(4), 697-711. https://doi.org/10.18187/pjsor.v16i4.3260

23. Elgohari, H. and Yousof, H. M. (2020b). New Extension of Weibull Distribution: Copula, Mathematical Properties and Data Modeling. Statistics, Optimization \& Information Computing, 8(4), 972-993. https://doi.org/10.19139/soic-2310-5070-1036

24. El-Morshedy, M., Alshammari, F. S., Hamed, Y. S., Eliwa, M. S., Yousof, H. M. (2021). A New Family of Continuous Probability Distributions. Entropy, 23, 194. https://doi.org/10.3390/e23020194

25. Farlie, D. J. G. (1960) The performance of some correlation coefficients for a general bivariate distribution. Biometrika, 47, 307-323

26. Gross, J. and Clark, V. A. (1975). Survival Distributions: Reliability Applications in the Biometrical Sciences, John Wiley, New York, USA.

27. Gumbel, E. J. (1961). Bivariate logistic distributions. Journal of the American Statistical Association, 56(294), 335-349.

28. Gumbel, E. J. (1960) Bivariate exponential distributions. Journ. Amer. Statist. Assoc., 55, 698-707. 
29. George, R. and Thobias, S. (2019). Kumaraswamy Marshall-Olkin exponential distribution. Communications in Statistics-Theory and methods, 48(8), 1920-1937.

30. Ghitany, M.E., Al-Hussaini, E.K., AlJarallah, R. A. (2005). Marshall-Olkin extended Weibull distribution and its application to censored data. J Appl Stat, 32:1025-1034.

31. Goual, H., Yousof, H. M. and Ali, M. M. (2019). Validation of the odd Lindley exponentiated exponential by a modified goodness of fit test with applications to censored and complete data. Pakistan Journal of Statistics and Operation Research, 745-771.

32. Goual, H. and Yousof, H. M. (2020). Validation of Burr XII inverse Rayleigh model via a modified chi-squared goodness-of-fit test. Journal of Applied Statistics, 47(3), 393-423.

33. Goual, H., Yousof, H. M. and Ali, M. M. (2020). Lomax inverse Weibull model: properties, applications, and a modified Chi-squared goodness-of-fit test for validation. Journal of Nonlinear Sciences \& Applications (JNSA), 13(6), 330-353.

34. Gross, J. and Clark, V. A. (1975). Survival Distributions: Reliability Applications in the Biometrical Sciences, John Wiley, New York, USA.

35. Hamedani, G. G., Altun, E, Korkmaz, M. C., Yousof, H. M. and Butt, N. S. (2018). A new extended G family of continuous distributions with mathematical properties, characterizations and regression modeling. Pak. J. Stat. Oper. Res., 14(3), 737-758.

36. Hamedani, G. G. Rasekhi, M., Najib, S. M., Yousof, H. M. and Alizadeh, M., (2019). Type II general exponential class of distributions Pak. J. Stat. Oper. Res., XV (2), 503-523.

37. Hamedani, G. G. Yousof, H. M., Rasekhi, M., Alizadeh, M., Najibi, S. M. (2017). Type I general exponential class of distributions. Pak. J. Stat. Oper. Res., XIV (1), 39-55.

38. Ibrahim, M., Altun, E. and Yousof, H. M. (2020). A new distribution for modeling lifetime data with different methods of estimation and censored regression modeling. Statistics, Optimization and Information Computing, 8(2), 610-630.

39. Ibrahim, M., Altun, E., Goual, H., and Yousof, H. M. (2020). Modified goodness-of-fit type test for censored validation under a new Burr type XII distribution with different methods of estimation and regression modeling. Eurasian Bulletin of Mathematics, 3(3), $162-182$.

40. Ibrahim. M., Handique, L., Chakraborty, S., Butt, N. S. and M. Yousof, H. (2021). A New Three-parameter Xgamma Fréchet Distribution with Different Methods of Estimation and Applications. Pakistan Journal of Statistics and Operation Research, 17(1), 291-308.

41. Ibrahim, M., Yadav, A. S., Yousof, H. M., Goual, H. and Hamedani, G. G. (2019). A new extension of Lindley distribution: modified validation test, characterizations and different methods of estimation. Communications for Statistical Applications and Methods, 26(5), 473-495.

42. Johnson, N. L. and Kotz, S. (1975) On some generalized Farlie-Gumbel-Morgenstern distributions. Commun. Stat. Theory, 4, 415427.

43. Johnson, N. L. and Kotz, S. (1977) On some generalized Farlie-Gumbel-Morgenstern distributions- II: Regression, correlation and further generalizations. Commun. Stat.Theory, 6, 485-496.

44. Khalil, M. G., Hamedani, G. G. and Yousof, H. M. (2019). The Burr X exponentiated Weibull model: Characterizations, mathematical properties and applications to failure and survival times data. Pakistan Journal of Statistics and Operation Research, 141-160.

45. Karamikabir, H., Afshari, M., Yousof, H. M., Alizadeh, M. and Hamedani, G. (2020). The Weibull Topp-Leone Generated Family of Distributions: Statistical Properties and Applications. Journal of The Iranian Statistical Society, 19(1), 121-161.

46. Korkmaz, M. C., Altun, E., Yousof, H. M. and Hamedani, G. G. (2020). The Hjorth's IDB Generator of Distributions: Properties, Characterizations, Regression Modeling and Applications. Journal of Statistical Theory and Applications, 19(1), 59-74.

47. Korkmaz, M. C. Yousof, H. M. and Hamedani G. G. (2018a). The exponential Lindley odd log-logistic G family: properties, characterizations and applications. Journal of Statistical Theory and Applications, 17(3), 554 - 571.

48. Korkmaz, M. C., Yousof, H. M., Hamedani G. G. and Ali, M. M. (2018b). The Marshall-Olkin generalized G Poisson family of distributions, Pakistan Journal of Statistics, 34(3), 251-267.

49. Korkmaz, M. C., Yousof, H. M., Hamedani G. G. and Ali, M. M. (2018). The Marshall-Olkin generalized G Poisson family of distributions, Pakistan Journal of Statistics, 34(3), 251-267.

50. Lee, C., Famoye, F. and Olumolade, O. (2007). Beta-Weibull distribution: some properties and applications to censored data. Journal of modern applied statistical methods, 6(1), 17.

51. Mansour, M. M., Ibrahim, M., Aidi, K., Shafique Butt, N., Ali, M. M., Yousof, H. M. and Hamed, M. S. (2020a). A New Log-Logistic Lifetime Model with Mathematical Properties, Copula, Modified Goodness-of-Fit Test for Validation and Real Data Modeling. Mathematics, 8(9), 1508.

52. Mansour, M. M., Butt, N. S., Ansari, S. I., Yousof, H. M., Ali, M. M. and Ibrahim, M. (2020b). A new exponentiated Weibull distribution's extension: copula, mathematical properties and applications. Contributions to Mathematics, 1 (2020) 57-66. DOI $10.47443 / \mathrm{cm} .2020 .0018$

53. Mansour, M., Korkmaz, M. C., Ali, M. M., Yousof, H. M., Ansari, S. I. and Ibrahim, M. (2020c). A generalization of the exponentiated Weibull model with properties, Copula and application. Eurasian Bulletin of Mathematics, 3(2), 84-102.

54. Mansour, M., Rasekhi, M., Ibrahim, M., Aidi, K., Yousof, H. M. and Elrazik, E. A. (2020d). A New Parametric Life Distribution with Modified Bagdonavičius-Nikulin Goodness-of-Fit Test for Censored Validation, Properties, Applications, and Different Estimation Methods. Entropy, 22(5), 592.

55. Mansour, M., Yousof, H. M., Shehata, W. A. and Ibrahim, M. (2020e). A new two parameter Burr XII distribution: properties, copula different estimation methods and modeling acute bone cancer data. Journal of Nonlinear Science and Applications, 13(5), 223-238.

56. Mansour, M. M., Butt, N. S., Yousof, H. M., Ansari, S. I. and Ibrahim, M. (2020f). A Generalization of Reciprocal Exponential Model: Clayton Copula, Statistical Properties and Modeling Skewed and Symmetric Real Data Sets. Pakistan Journal of Statistics and Operation Research, 16(2), 373-386.

57. Marshall, A. W. and Olkin, I. (1997). A new method for adding a parameter to a family of distributions with application to the Exponential and Weibull families. Biometrika, 84, 641-652.

58. Maurya, S. K. and Nadarajah, S. (1998). Poisson Generated Family of Distributions: A Review. Sankhya B, 1-57. 
59. Merovci, F., Alizadeh, M., Yousof, H. M. and Hamedani G. G. (2017). The exponentiated transmuted-G family of distributions: theory and applications, Communications in Statistics-Theory and Methods, 46(21), 10800-10822.

60. Merovci, F., Yousof, H. M. and Hamedani, G. G. (2020). The Poisson Topp Leone Generator of Distributions for Lifetime Data: Theory, Characterizations and Applications. Pakistan Journal of Statistics and Operation Research, 16(2), 343-355.

61. Nascimento, A. D. C., Silva, K. F., Cordeiro, G. M., Alizadeh, M. and Yousof, H. M. (2019). The odd Nadarajah-Haghighi family of distributions: properties and applications. Studia Scientiarum Mathematicarum Hungarica, 56(2), 1-26.

62. Pougaza, D. B. and Djafari, A. (2011). Maximum entropies copulas. In AIP Conference Proceedings (Vol. 1305, No. 1, pp. 329-336). American Institute of Physics.

63. Rezaei, S., B. B. Sadr, M. Alizadeh, and S. Nadarajah. (2017). Topp-Leone generated family of distributions: Properties and applications. Communications in Statistics: Theory and Methods 46 (6), 2893-2909.

64. Rodriguez-Lallena, J. A. and Ubeda-Flores, M. (2004). A new class of bivariate copulas. Statistics and Probability Letters, 66, 31525. Salman S., M. and Prayoto, S. (1999). Total time on test plot analysis for mechanical components of the RSG-GAS reactor. Atom Indones, 25(2), 155-61.

65. Yadav, A. S., Goual, H., Alotaibi, R. M., Ali, M. M. and Yousof, H. M. (2020). Validation of the Topp-Leone-Lomax model via a modified Nikulin-Rao-Robson goodness-of-fit test with different methods of estimation. Symmetry, 12(1), 57.

66. Yousof, H. M., Afify, A. Z., Alizadeh, M., Butt, N. S., Hamedani, G. G. and Ali, M. M. (2015). The transmuted exponentiated generalized-G family of distributions, Pak. J. Stat. Oper. Res., 11, 441-464.

67. Yousof, H. M., Afify, A. Z., Alizadeh, M., Hamedani G. G., Jahanshahi, S. M. A., Ghosh, I., (2018a). The generalized transmuted Poisson-G family of Distributions, Pak. J. Stat. Oper. Res., Vol. 14 (4), pp. 759-779.

68. Yousof, H. M., Afify, A. Z., Hamedani, G. G. and Aryal, G. (2017a). The Burr X generator of distributions for lifetime data. Journal of Statistical Theory and Applications, 16, 288-305.

69. Yousof, H. M., Ali, M. M., Goual, H. and Ibrahim. M. (2021). A new reciprocal Rayleigh extension: properties, copulas, different methods of estimation and modified right censored test for validation, STATISTICS IN TRANSITION new series, forthcoming.

70. Yousof, H. M., Alizadeh, M., Jahanshahi, S. M. A., Ramires, T. G., Ghosh, I. and Hamedani, G. G. (2017b). The transmuted ToppLeone G family of distributions: theory, characterizations and applications. Journal of Data Science, 15(4), 723-740.

71. Yousof, H. M., Altun, E., Ramires, T. G., Alizadeh, M. and Rasekhi, M. (2018b). A new family of distributions with properties, regression models and applications, Journal of Statistics and Management Systems, 21(1), 163-188.

72. Yousof, H. M., Mansoor, M. Alizadeh, M., Afify, A. Z., Ghosh, I. and Afify, A. Z. (2020). The Weibull-G Poisson family for analyzing lifetime data. Pak. J. Stat. Oper. Res., 16 (1), 131-148. 